AdVANCES IN COMBINATORICS, 2021:8, 28 pp.

www.advancesincombinatorics.com

\title{
Improved Bounds for Centered Colorings
}

\author{
Michał Dębski Stefan Felsner* Piotr Micek ${ }^{\dagger} \quad$ Felix Schröder
}

Received 25 August 2020; Published 16 August 2021

\begin{abstract}
A vertex coloring $\phi$ of a graph $G$ is $p$-centered if for every connected subgraph $H$ of $G$ either $\phi$ uses more than $p$ colors on $H$ or there is a color that appears exactly once on $H$. Centered colorings form one of the families of parameters that allow to capture notions of sparsity of graphs: A class of graphs has bounded expansion if and only if there is a function $f$ such that for every $p \geq 1$, every graph in the class admits a $p$-centered coloring using at most $f(p)$ colors.

In this paper, we give upper bounds for the maximum number of colors needed in a $p$-centered coloring of graphs from several widely studied graph classes. We show that: (1) planar graphs admit $p$-centered colorings with $\mathcal{O}\left(p^{3} \log p\right)$ colors where the previous bound was $\mathcal{O}\left(p^{19}\right)$; (2) bounded degree graphs admit $p$-centered colorings with $\mathcal{O}(p)$ colors while it was conjectured that they require an exponential number of colors in $p$; (3) graphs avoiding a fixed graph as a topological minor admit $p$-centered colorings with a polynomial in $p$ number of colors. All these upper bounds imply polynomial algorithms for computing the colorings.

Prior to this work there were no non-trivial lower bounds known. We show that: (4) there are graphs of treewidth $t$ that require $\left(\begin{array}{c}p+t \\ t\end{array}\right)$ colors in any $p$-centered coloring; this matches the known upper bound. (5) there are planar graphs that require $\Omega\left(p^{2} \log p\right)$ colors in any $p$-centered coloring. We also give asymptotically tight bounds for outerplanar graphs and planar graphs of treewidth 3 . We prove our results using a variety of techniques. The upper bound for planar graphs involves an application of a recent structure theorem while the upper bound for bounded degree graphs comes from the entropy compression method. We lift the result for bounded degree graphs to graphs avoiding a fixed topological minor using the Grohe-Marx structure theorem.
\end{abstract}

A preliminary version of this paper appeared as an extended abstract in the Proceedings of the 31st annual ACM-SIAM Symposium on Discrete Algorithms, SODA 2020 [1].

*Supported by DFG grant FE 340/13-1.

† Supported partially by a Polish National Science Center grant (BEETHOVEN; UMO-2018/31/G/ST1/03718). 


\section{Introduction}

Structural graph theory has expanded beyond the study of classes of graphs that exclude a fixed minor. One of the driving forces was, and is, to develop efficient algorithms for computationally hard problems for graphs that are 'structurally sparse'. Nešetřil and Ossona de Mendez introduced the concepts of classes of graphs with bounded expansion [16] and classes of graphs that are nowhere dense [17]. These are very robust properties that include every class excluding a fixed minor but also graphs of bounded book-thickness or graphs that allow drawings with bounded number of crossings per edge, see [19]. At first sight, bounded expansion might seem to be a weak property for a graph class. Yet, this notion captures enough structure to allow solving a wide range of algorithmic problems efficiently: Dvořák, Král' and Thomas [5] devised an FPT algorithm for testing first-order definable properties in classes of bounded expansion.

One reason that these new notions attracted much attention is the realization that they can be characterized in several, seemingly different ways. Instead of providing the original definition we define bounded expansion in terms of centered chromatic numbers.

\subsection{Centered colorings}

Let $p$ be a positive integer. A vertex coloring $\phi$ of a graph $G$ is $p$-centered if for every connected subgraph $H$ of $G$ either $\phi$ uses more than $p$ colors on $H$ or there is a color that appears exactly once on $H$. The p-centered chromatic number $\chi_{p}(G)$ of $G$ is the minimum integer $k$ such that there is a $p$-centered coloring of $G$ using $k$ colors.

A vertex coloring of a graph is 1-centered if and only if it is proper, i.e., adjacent vertices receive distinct colors. A vertex coloring is 2-centered if and only if it is a star coloring, i.e., it is proper and every path on four vertices receives at least three colors. A class $\mathcal{C}$ of graphs is of bounded expansion if and only if there exists a function $f: \mathbb{N} \rightarrow \mathbb{N}$ such that for every integer $p \geq 1$ and every $G \in \mathcal{C}$ we have $\chi_{p}(G) \leq f(p)$. Nešetřil and Ossona de Mendez, who originally defined bounded expansion in terms of maximum densities of shallow minors, showed in [16] that the definitions are equivalent.

Colorings, such that the condition depending on $p$ is omitted, are called centered colorings. The centered chromatic number is the minimum number of colors in such a coloring and coincides with the treedepth for every graph $G$ ([18], Lemma 4.2). The treedepth of a graph $G$ is the minimum height of a rooted forest $F$ such that $G \subseteq \operatorname{clos}(F)$ where (1) a rooted forest is a disjoint union of rooted trees; (2) the height of a rooted forest $F$ is maximum number of vertices on a path from a root to a leaf in $F$; (3) the closure $\operatorname{clos}(F)$ of $F$ is the graph with vertex set $V(F)$ and edge set $\{\{v, w\} \mid v$ is an ancestor of $w$ in $F\}$. As observed in [18], every $p$-centered coloring of a graph $G$ is also a treedepth- $p$ coloring of $G$, i.e., for every $i \leq p$ the union of any $i$ color classes induces a subgraph of $G$ of treedepth at most $i$.

\subsection{Algorithmic applications}

Low treedepth colorings are a central tool for designing parametrized algorithms in classes of bounded expansion. For example, Pilipczuk and Siebertz [21] showed that when $\mathcal{C}$ is a class of graphs avoiding a fixed minor, then it can be decided whether a given $p$-vertex graph $H$ is a subgraph of a given $n$-vertex graph $G$ in $\mathcal{C}$ in time $2^{\mathcal{O}(p \log p)} \cdot n^{\mathcal{O}(1)}$ and space $n^{\mathcal{O}(1)}$. The algorithm witnessing this statement starts with 


\section{IMPROVED BOUNDS FOR CENTERED COLORINGS AUTHOR}

a computation of a $p$-centered coloring of $G$ with $p^{\mathcal{O}(1)}$ colors, and for each $p$-tuple of colors it applies a procedure to solve this problem for graphs $G$ of treedepth at most $p$. The results of our paper imply a corresponding algorithm for the case where $\mathcal{C}$ is a class of graphs avoiding a fixed graph as a topological minor.

The polynomial space complexity mentioned above is remarkable. Typically dynamic programming algorithms on tree decompositions use space exponential in the width of the decomposition and there are complexity-theoretical reasons to believe that without significant increase in time complexity, this cannot be avoided. On the other hand treedepth decompositions, sometimes called elimination trees, allow to devise algorithms using only polynomial space in the height of the decomposition, see a thorough study of this phenomenon by Pilipczuk and Wrochna [22].

Clearly, the running times of algorithms based on $p$-centered colorings heavily depend on the number of colors used. A recent experimental work by O'Brien and Sullivan [20] points to the lack of efficient coloring procedures as the major bottleneck for applicability of these algorithms in practice.

\subsection{Results}

In this paper, we improve the bounds for the maximum $\chi_{p}(G)$ for graphs $G$ in several important sparse classes of graphs. Most importantly, we reduce the upper bound on the number of colors needed for a $p$-centered coloring of planar graphs from $\mathcal{O}\left(p^{19}\right)$ to $\mathcal{O}\left(p^{3} \log p\right)$ and we show how to color graphs of bounded degree with $\mathcal{O}(p)$ colors; it was previously believed that there was an exponential lower bound for this latter class. All our bounds are supported with polynomial time algorithms computing the coloring.

We proceed with a presentation of our results.

Theorem 1. Planar graphs admit p-centered colorings with $\mathcal{O}\left(p^{3} \log p\right)$ colors.

The previously best known bound was $\mathcal{O}\left(p^{19}\right)$ due to Pilipczuk and Siebertz [21]. A key tool responsible for the improvement of the exponent is a recent structure theorem for planar graphs due to Dujmović et al. [3] which has its roots in [21]. In Section 2, we give a precise statement of the theorem and we show how to use it to color a planar graph in a $p$-centered way with $\mathcal{O}(p) \cdot f(p)$ colors where $f(p)$ is the maximum number of colors needed in a $p$-centered coloring of planar graphs of treewidth at most 3 . The $\mathcal{O}\left(p^{3} \log p\right)$ bound then follows from Theorem 6.(iii).

Next we conclude an improved bound for graphs drawn on surfaces with bounded genus.

Theorem 2. Graphs with Euler genus g admit p-centered colorings with $\mathcal{O}\left(g p+p^{3} \log p\right)$ colors.

The previous best known bound was $\mathcal{O}\left(g^{2} p^{3}+p^{19}\right)$, see [21]. Our result follows from the bound for planar graphs combined with a product structure theorem from [3].

Graphs with bounded maximum degree are sparse but somehow much less structured than planar graphs, e.g. they allow any graph as a minor. It was conjectured in [21], that the number of colors needed for $p$-centered colorings in the class of graphs of maximum degree 3 is exponential in $p$. This was supported by similar bounds for weak coloring numbers which is another family of parameters capturing the notion of bounded expansion. We disprove the conjecture by providing an upper bound which is linear in $p$. 
Theorem 3. Graphs with bounded degree admit p-centered colorings with $\mathcal{O}(p)$ colors. More specifically, for every $p \geq 1$ and every graph $G$ with maximum degree at most $\Delta$ :

$$
\chi_{p}(G) \in \mathcal{O}\left(\Delta^{2-1 / p} \cdot p\right) .
$$

Dubois et al. [2] recently proved an almost matching lower bound. By analyzing an appropriate random graph they show that there are graphs of maximum degree $\Delta$ that require $\Omega\left(\Delta^{2-1 / p} \cdot p \cdot \ln ^{-1 / p} \Delta\right)$ colors in any $p$-centered coloring.

We prove Theorem 3 with an entropy compression type argument. The idea is to run a naïve randomized algorithm for coloring a graph in the $p$-centered way. Assuming that this algorithm fails on a long run, for every possible evaluation of random experiments, we can compress a random string of bits below the entropy bound. The contradiction shows that there must be a sequence of bits which leads to a successful run. The entropy compression method is inspired by the algorithmic proof of the Lovász local lemma by Moser and Tardos [15]. An instructive overview of the method can be found at Tao's blog [24] or in a paper by Grytczuk, Kozik, and Micek [8] where nonrepetitive sequences over a finite alphabet are constructed. In the case $p=2$, i.e., for star chromatic number, our argument matches the upper bound $\mathcal{O}\left(\Delta^{1.5}\right)$ by Fertin, Raspaud, and Reed [6].

One of the strong results from [21] is that graphs excluding a fixed minor admit $p$-centered colorings with a polynomial in $p$ number of colors. The proof goes through the graph minor structure theorem by Robertson and Seymour [23]. Grohe and Marx [7] extended the structure theorem to graphs avoiding a fixed topological minor. In some sense, they showed that incorporating the bounded-degree graphs into the structural decomposition given in [23] is enough to obtain an approximate characterization for classes excluding a fixed topological minor. For these reasons, we have been able to lift our result for bounded degree graphs and obtain the following general statement.

Theorem 4. For every graph $H$ there is a polynomial $f$ such that the graphs excluding $H$ as a topological minor admit p-centered colorings with at most $f(p)$ colors.

Prior to our work there were no non-trivial lower bounds for the maximum number of colors required in a $p$-centered coloring. The lower bounds for planar graphs, graphs with bounded treewidth and even graphs excluding a fixed minor were only linear in $p$. We present constructions forcing $\chi_{p}(G)$ to be superlinear in $p$.

Theorem 5. For every $p \geq 0$ and $t \geq 0$, there is a graph $G$ of treewidth at most $t$ with

$$
\chi_{p}(G) \geq\left(\begin{array}{c}
p+t \\
t
\end{array}\right) .
$$

This lower bound is sharp as Pilipczuk and Siebertz [21] showed that for every $p \geq 1$, every $t \geq 1$, every graph $G$ of treewidth at most $t$ has $\chi_{p}(G) \leq\left(\begin{array}{c}p+t \\ t\end{array}\right)$. In fact, we show a slightly stronger statement than Theorem 5 concerning a relaxation of $p$-centered colorings, namely the $p$-linear colorings introduced in [12]. See the precise statement in Section 6.

Finally, we present asymptotically tight lower and upper bounds for the maximum $p$-centered chromatic numbers of outerplanar graphs and planar graphs of treewidth at most 3, i.e., subgraphs of stacked triangulations (see [11] for more details). In particular Theorem 6.(iv) implies the best known lower bound $\Omega\left(p^{2} \log p\right)$ for the maximum $p$-centered chromatic number of planar graphs. 


\section{Theorem 6.}

(i) Outerplanar graphs admit p-centered colorings with $\mathcal{O}(p \log p)$ colors.

(ii) There is a family of outerplanar graphs that requires $\Omega(p \log p)$ colors in any $p$-centered coloring.

(iii) Planar graphs of treewidth at most 3, i.e., subgraphs of stacked triangulations, admit p-centered colorings with $\mathcal{O}\left(p^{2} \log p\right)$ colors.

(iv) There is a family of planar graphs of treewidth 3 that requires $\Omega\left(p^{2} \log p\right)$ colors in any $p$-centered coloring.

It turns out that the lower bounds (ii) and (iv) and the upper bounds (i) and (iii) of Theorem 6 are initial steps of a more general result stated in Theorem 7. The statement of the theorem involves the notion of simple treewidth which will be formally introduced in Subsection 5.2, for the moment we just remark that graphs of simple treewidth at most 2 are outerplanar graphs and graphs of simple treewidth at most 3 are planar graphs of treewidth at most 3 .

\section{Theorem 7.}

(i) Graphs of simple treewidth at most $k$ admit p-centered colorings with $\mathcal{O}\left(p^{k-1} \log p\right)$ colors.

(ii) There is a family of graphs of simple treewidth $k$ that requires $\Omega\left(p^{k-1} \log p\right)$ colors in any $p$ centered coloring.

\subsection{Paper Overview}

In Section 2, we present a new structure theorem for planar graphs from [3] and show how to apply it to get good bounds for $\chi_{p}(G)$ when $G$ is planar or when $G$ has bounded genus. In Section 3, we set up an entropy compression argument to prove an $\mathcal{O}(p)$ upper bound for graphs with bounded degree. In Section 4 , we lift the previous result to obtain a polynomial bound for graphs avoiding a fixed graph as a topological minor. In Sections 5 and 7, we give the upper bounds and lower bounds, respectively, for graphs of bounded simple treewidth. Section 6 is devoted to the lower bound for graphs of bounded treewidth.

Following the presentation of each upper bound, we give a short justification why the proof gives a polynomial time algorithm computing the actual coloring.

\section{Upper bounds for planar graphs: An application of the product struc- ture theorem}

This section is devoted to the proof of our upper bounds for planar graphs and graphs with bounded genus. The proof is based on results for stacked triangulations, in particular Theorem 6(iii).

We need some additional notation: By $G \otimes H$ we denote the graph with vertex set $V(G) \times V(H)$ which has an edge between $(u, w)$ and $\left(u^{\prime}, w^{\prime}\right)$ if $u u^{\prime} \in E(G)$ and $w w^{\prime} \in E(H)$, or $u=u^{\prime}$ and $w w^{\prime} \in E(H)$, or $u u^{\prime} \in E(G)$ and $w=w^{\prime}$.

Let $G^{p}$ be the $p$ th power of $G$, i.e., a pair $u w$ is an edge of $G^{p}$ whenever the distance between $u$ and $w$ in $G$ is at most $p$. Note that any proper coloring of $G^{p}$ is a $p$-centered coloring of $G$, because any two vertices of the same color have distance at least $p+1$ in $G$ and the $p+1$ first vertices on a path from one of these vertices to the other form a clique in $G^{p}$, thus they receive pairwise different colors. Actually, this 
shows that these colorings admit an even stronger condition than $p$-centered colorings: Any connected subgraph $H$ in such a coloring either contains more than $p$ colors or it contains every color at most once. We will use this property at the end of the proof of the following lemma.

\section{Lemma 8.}

$$
\chi_{p}\left(H_{1} \otimes H_{2}\right) \leq \chi_{p}\left(H_{1}\right) \cdot \chi\left(H_{2}^{p}\right)
$$

Proof. Let $\psi_{1}$ be a $p$-centered coloring of $H_{1}$ and $\psi_{2}$ be a proper coloring of $H_{2}^{p}$. With $\pi_{1}$ and $\pi_{2}$ we denote the the projections from $V\left(H_{1}\right) \times V\left(H_{2}\right)$ to its components. We claim that the product coloring $\phi(v, w)=\left(\psi_{1}(v), \psi_{2}(w)\right)$ is a $p$-centered coloring of $G=H_{1} \otimes H_{2}$. Consider a connected subgraph $G^{\prime}$ of $G$. From the definition of $\otimes$ it follows that $H_{1}^{\prime}:=\pi_{1}\left(G^{\prime}\right)$ is connected. Since $\psi_{1}$ is a $p$-centered coloring of $H_{1}$, it either uses more than $p$ colors on $H_{1}^{\prime}$ or there is a vertex $\hat{v} \in H_{1}^{\prime}$, whose color is unique in $H_{1}^{\prime}$. In the first case, $\phi$ uses more than $p$ colors on $G^{\prime}$ and we are done. In the second case consider a vertex $(\hat{v}, w) \in G^{\prime}$. The color of $(\hat{v}, w)$ is unique in $G^{\prime}$ unless there is a $w^{\prime} \in H_{2}$ with $w \neq w^{\prime}, \psi_{2}(w)=\psi_{2}\left(w^{\prime}\right)$, and $\left(\hat{v}, w^{\prime}\right) \in G^{\prime}$. If such a vertex $w^{\prime}$ exists, then let $P$ be a path in $G^{\prime}$ from $(\hat{v}, w)$ to $\left(\hat{v}, w^{\prime}\right)$. Since not all colors in $\pi_{2}(P)$ are unique and $\pi_{2}(P)$ is connected in $H_{2}, \psi_{2}$ uses more than $p$ colors on $\pi_{2}(P)$, whence $\phi$ uses more than $p$ colors on $P \subset G^{\prime}$.

Let $G+H$ denote the complete join of the graphs $G$ and $H$, this is the graph whose vertex set is the disjoint union $V(G) \cup V(H)$ the edge set contains $E(G)$ and $E(H)$ together all possible edges with one end in $V(G)$ and one in $V(H)$. The following theorem is due to Dujmović et al. [3].

Theorem 9 ([3], Theorem 37). Every graph of Euler genus $g$ is a subgraph of:

1. $H \otimes P \otimes K_{\max (2 g, 3)}$ for some apex graph $H$ of treewidth at most 4 and $a$ path $P$.

2. $\left(K_{2 g}+H_{8}\right) \otimes P$ for some planar graph $H_{8}$ of treewidth at most 8 and a path $P$.

The proof given in [3] actually reveals that the graph $H$ in Theorem 9(1) is an apex graph over a stacked triangulation, i.e., removing the apex leaves a planar graph $H^{\prime}$ with treewidth at most 3 . Hence, with Theorem 6(iii) we get

$$
\chi_{p}(H) \leq \chi_{p}\left(H^{\prime}\right)+1 \in \mathcal{O}\left(p^{2} \log p\right) .
$$

Corollary 10. For any planar graph $G$ and any graph $G_{g}$ of Euler genus $g$, we have:

$$
\begin{aligned}
\chi_{p}(G) & \in \mathcal{O}\left(p^{3} \log p\right), \\
\chi_{p}\left(G_{g}\right) & \in \mathcal{O}\left(g p^{3} \log p\right), \\
\chi_{p}\left(G_{g}\right) & \in \mathcal{O}\left(g p+p^{4} \log p\right) .
\end{aligned}
$$

Proof. We begin with three easy observations. The $p$ th power of the complete graph is just the complete graph, in particular $\chi\left(K_{n}^{p}\right)=\chi\left(K_{n}\right)=n$. A periodic coloring of a path $P$ with $p+1$ colors shows that $\chi\left(P^{p}\right)=p+1$. Furthermore, the additon of an apex to a graph can increase its $p$-centered chromatic number by at most 1 . With Theorem 9 it now follows that

$$
\begin{aligned}
\chi_{p}(G) & \leq \chi_{p}\left(H \otimes P \otimes K_{3}\right) \leq \chi_{p}(H \otimes P) \cdot \chi\left(K_{3}^{p}\right) \leq \chi_{p}(H) \cdot \chi\left(P^{p}\right) \cdot 3 \in \mathcal{O}\left(p^{3} \log p\right) \\
\chi_{p}\left(G_{g}\right) & \leq \chi_{p}\left(H \otimes P \otimes K_{2 g+3}\right) \leq \chi_{p}(H) \cdot \chi\left(P^{p}\right) \cdot(2 g+3) \in \mathcal{O}\left(g p^{3} \log p\right) \\
\chi_{p}\left(G_{g}\right) & \leq \chi_{p}\left(K_{2 g}+H_{8}\right) \cdot \chi\left(P^{p}\right) \leq\left(2 g+\chi_{p}\left(H_{8}\right)\right) \cdot(p+1) \in \mathcal{O}\left(g p+p^{4} \log p\right)
\end{aligned}
$$




\section{IMPROVED BOUNDS FOR CENTERED COLORINGS AUTHOR}

For the final inequality of the first two lines we have used $(*)$. In the last line, we used that $H_{8}$ is planar and therefore by the first line has a $p$-centered coloring using at most $\mathcal{O}\left(p^{3} \log p\right)$ colors.

A coloring procedure which follows the proof of Theorem 9 more closely can be used to show that graphs of Euler genus $g$ actually admit $p$-centered colorings with $\mathcal{O}\left(g p+p^{3} \log p\right)$ colors. The proof for this slightly improved bound has been detailed in the conference version of this paper [1]. There it is also indicated how the proofs can be turned into quadratic time algorithms for colorings which respect the given bounds for the number of colors. The bottleneck of the coloring algorithm is the computation of the product structure. Recently Morin [14] has shown that a $\left(K_{2 g}+H_{8}\right) \otimes P$ structure can be constructed in $\mathcal{O}(n \log n)$ time, this yields a corresponding improvement in the running time of the coloring algorithm.

\section{Upper bound for bounded degree: The entropy compression method}

In this section we prove Theorem 3 .

Let $p$ be a positive integer and let $G$ be a graph with maximum degree at most $\Delta$. Fix an arbitrary ordering $\Pi=\left(v_{1}, \ldots, v_{n}\right)$ of $V(G)$. For every vertex $v \in V(G)$, fix an arbitrary ordering of the edges adjacent to $v$. Let $c=\left\lceil 2^{10} \cdot \Delta^{2-1 / p} \cdot p\right\rceil$ and let $M$ be a sufficiently large integer divisible by $2 p$.

A partial coloring of $G$ is a function $f: V(G) \rightarrow C \cup\{\diamond\}$ where $C$ is a set of colors used by $f$ and $\diamond$ is an extra value indicating that no color is assigned. A vertex $v$ with $f(v) \in C$ is said to be colored by $f$ while a vertex $v$ with $f(v)=\diamond$ is uncolored by $f$. A connected subgraph $H$ of $G$ is a violator with respect to a partial coloring $f$ if:

1. all vertices in $H$ are colored by $f$;

2. $f$ uses at most $p$ colors on $H$; and

3. no color is unique in $H$ under $f$.

Algorithm 1 below is a naïve randomized algorithm which tries to color $G$ in a $p$-centered fashion with $c$ colors. The algorithm starts with all vertices uncolored. In every step, the first uncolored vertex from $\Pi$ is colored with a random color from $\{1, \ldots, c\}$. If this new assignment creates a violator $H$, then $\min (|V(H)|, 2 p)$ vertices in $H$ are uncolored, including the vertex colored in this step. (One could erase the colors of all the vertices in $H$ and get a $\mathcal{O}\left(\Delta^{2} p\right)$ bound; we present this slightly more involved version to get $\mathcal{O}\left(\Delta^{2-1 / p} p\right)$.)

To trade probabilistic arguments for counting we replace random sampling by a prescribed sequence $X=\left(x_{1}, \ldots, x_{M}\right)$ of colors, where $x_{i} \in[c]$ for every $i \in[M]$, and take $X$ as part of the input of the algorithm, see Algorithm 1.

If the algorithm breaks the loop at line 4 and outputs a coloring $f$, then $f$ is a $p$-centered coloring of $G$ using at most $c$ colors. Indeed, we keep an invariant that after each iteration there is no violator with respect to $f$. This is obviously true before the first iteration. Now within step $i$, the newly colored vertex $w_{i}$ could create violators, but each of them must contain $w_{i}$. When the algorithm uncolors some vertices in a single violator, it always uncolors $w_{i}$ itself. So no violators remain.

We define a structure called $\operatorname{LOG}(X)$ which accumulates data during the execution of the algorithm. We will show that unless input $X$ leads to a break we can reconstruct $X$ from $\log (X)$, i.e., the map 


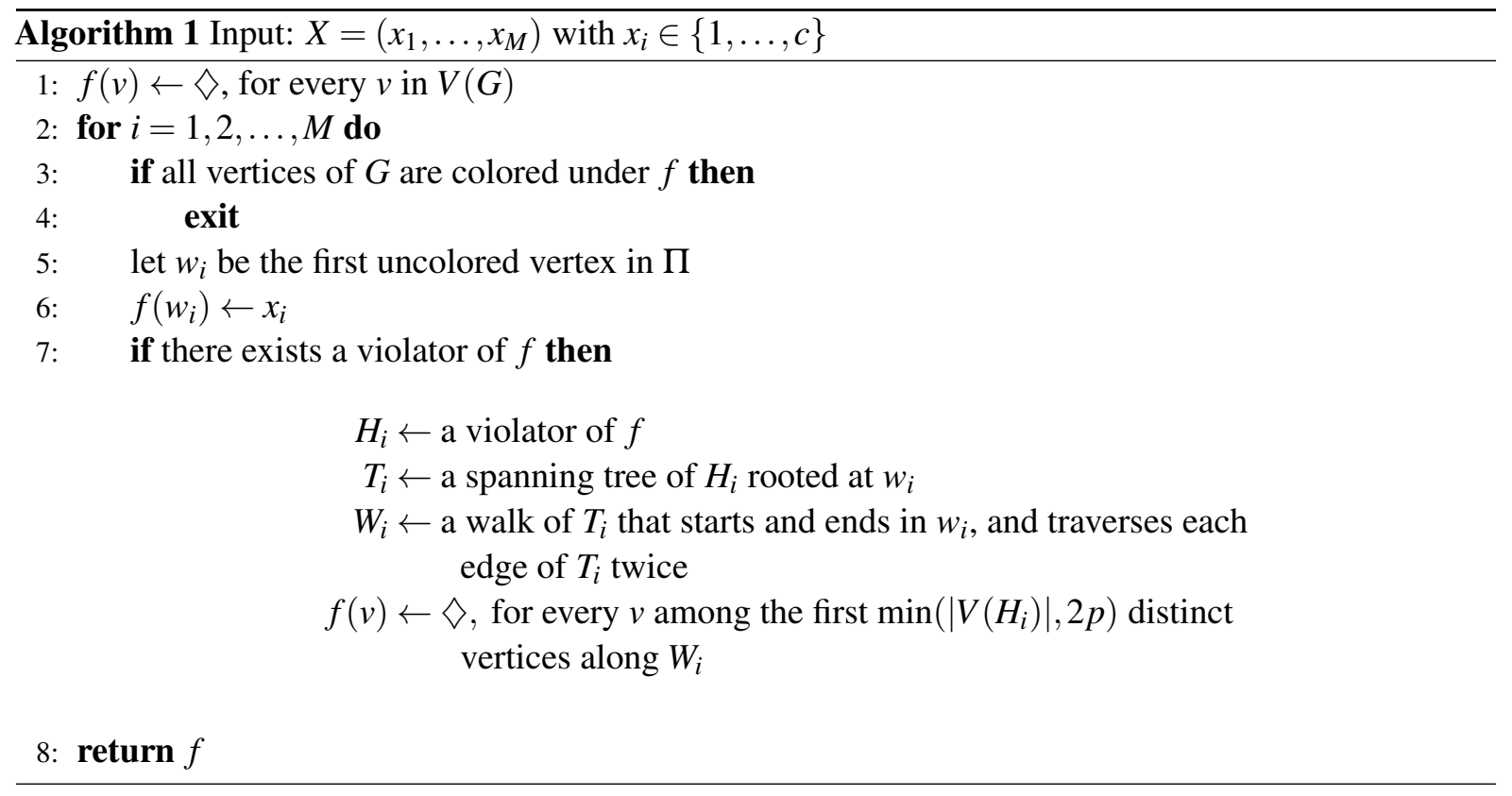

$X \mapsto \operatorname{LOG}(X)$ is injective. On the other hand, we will show that $\log (X)$ takes strictly less than $c^{M}$ values. This is a contradiction as there is no injective map from a set of size $c^{M}$ into a smaller one. Thus there has to be an input which leads to a break (line 4), i.e., to a valid coloring.

We continue to define LOG and to show how to reconstruct $X$ from $\operatorname{LOG}(X)$ when the algorithm with input $X$ does not break at line 4 .

The data in $\operatorname{LOG}(X)$ is a tuple $(Z, \Sigma, \Gamma, F)$, the individual components of $\operatorname{LOG}(X)$ are described in the following.

In $Z=\left(z_{0}, z_{1}, \ldots, z_{M}\right)$ we record the number of colored vertices during the process. Specifically, $z_{i}$ is the number of colored vertices after finishing the $i$-th step of the loop. Note that $z_{0}=0, z_{1}=1$ and for $i>1$ either $z_{i}=z_{i-1}+1$ or $z_{i}=z_{i-1}+1-\min \left(h_{i}, 2 p\right)$, where $h_{i}=\left|V\left(H_{i}\right)\right|$ is the number of vertices of the violator $H_{i}$.

In $\Sigma$ we collect information about the violator subgraphs $H$ which get partially uncolored at some iteration of the loop.

Suppose that in the $i$-th step of the algorithm a subgraph $H_{i}$ is recognized as a violator. Let $T_{i}$ and $W_{i}$ be the structures fixed by the algorithm after line 7, so $T_{i}$ is a spanning tree of $H_{i}$ rooted at $w_{i}$ and $W_{i}=\left(u_{0}, u_{1}, \ldots, u_{2 h_{i}-2}\right)$ is a walk in $G$ traversing $T_{i}$ such that the walk starts and ends at $w_{i}$, i.e., $u_{0}=u_{2 h_{i}-2}=w_{i}$, and each edge of $T_{i}$ is traversed twice. Let $W_{i}^{\prime}$ be the walk in $G$ going along $W_{i}$ until $W_{i}$ has visited $m_{i}=\min \left(\left|V\left(H_{i}\right)\right|, 2 p\right)$ distinct vertices and after that $W_{i}^{\prime}$ takes the direct path in $T_{i}$ back to the root $w_{i}$ where it ends. If $\left|V\left(H_{i}\right)\right| \leq 2 p$, then $W_{i}^{\prime}=W_{i}$, otherwise $W_{i}^{\prime}$ is a walk travering a subtree $T_{i}^{\prime}$ of $T_{i}$ with $2 p$ vertices. In either case the traversed tree has $m_{i}-1$ edges, whence $W_{i}^{\prime}$ has length $2 m_{i}-2$. Let $\left(u_{0}^{\prime}, u_{1}^{\prime}, \ldots, u_{2 m_{i}-2}^{\prime}\right)$ be the ordered list of vertices visited by $W_{i}^{\prime}$. For $j \in\left\{0, \ldots, 2 m_{i}-3\right\}, u_{j}^{\prime} u_{j+1}^{\prime}$ is a forward step if $u_{j}^{\prime}$ is the parent of $u_{j+1}^{\prime}$ in $T_{i}$. Otherwise, $u_{j+1}^{\prime}$ is the parent of $u_{j}^{\prime}$ in $T_{i}$ and $u_{j}^{\prime} u_{j+1}^{\prime}$ is a backward step. 


\section{IMPROVED BOUNDS FOR CENTERED COLORINGS AUTHOR}

We encode $W_{i}^{\prime}$ in two lists. The binary list $B_{i}=\left(b_{0}, b_{1}, b_{2}, \ldots, b_{2 m_{i}-3}\right)$ records which steps of $W_{i}^{\prime}$ are forward and which are backward, i.e., $b_{j}=1$ if $u_{j}^{\prime} u_{j+1}^{\prime}$ is a forward step and $b_{j}=0$ if $u_{j}^{\prime} u_{j+1}^{\prime}$ is backward. Clearly, $m_{i}-1$ steps are forward and $m_{i}-1$ steps are backward. The list $L_{i}=\left(\ell_{1}, \ldots, \ell_{m_{i}-1}\right)$ with $\ell_{k} \in\{1, \ldots, \Delta\}$ collects data about the forward steps of $W_{i}^{\prime}$. If $u_{j}^{\prime} u_{j+1}^{\prime}$ is the $k$-th forward step in $W_{i}^{\prime}$, then $\ell_{k}$ is the unique number such that $u_{j}^{\prime} u_{j+1}^{\prime}$ is the $\ell_{k}$-th edge in the fixed ordering of edges adjacent to $u_{j}^{\prime}$.

Initially $\Sigma=(B, L)$ consists of two empty lists, and whenever it comes to uncoloring a violator $H_{i}$ in the course of the algorithm then $B_{i}$ is appended to $B$ and $L_{i}$ is appended to $L$.

In $\Gamma$ we collect information about the colors of the vertices of violator subgraphs at the moment they are uncolored within the iteration process.

Again, suppose that in the $i$-th step of the algorithm a subgraph $H_{i}$ with $h_{i}=\left|V\left(H_{i}\right)\right|$ is partially uncolored, and let $m_{i}=\min \left(h_{i}, 2 p\right)$. Let $W_{i}^{\prime}$ be the traversal of a subgraph of $H_{i}$ fixed in the definitions of $B_{i}$ and $L_{i}$. Let $V_{i}=\left(v_{1}^{\prime}, \ldots, v_{m_{i}}^{\prime}\right)$ be the vertices of $W_{i}^{\prime}$ sorted by their first-appearance in $W_{i}^{\prime}$. Let $B_{i}^{\prime}=\left(b_{1}^{\prime}, \ldots, b_{m_{i}}^{\prime}\right)$ be a binary sequence such that $b_{i}^{\prime}=1$ if $f\left(v_{i}^{\prime}\right) \notin\left\{f\left(v_{1}^{\prime}\right), \ldots, f\left(v_{i-1}^{\prime}\right)\right\}$ and $b_{i}^{\prime}=0$ otherwise. Let $c_{i}=\sum b_{j}^{\prime}$ that is $c_{i}$ is the number of colors used by $f$ at $W_{i}^{\prime}$ before it is uncolored. Since $V\left(W_{i}^{\prime}\right) \subseteq V\left(H_{i}\right)$ and $H_{i}$ is a violator we have $c_{i} \leq p$. Let $d_{i}=m_{i}-c_{i}$. We claim that $d_{i} \geq c_{i}$. Indeed, if $h_{i}>2 p$ then $d_{i}=m_{i}-c_{i}=2 p-c_{i} \geq p$. Otherwise, $m_{i}=\left|V\left(H_{i}\right)\right|$ and since $H_{i}$ is a violator every color in $H_{i}$ is used at least twice so $d_{i}=h_{i}-c_{i} \geq c_{i}$ as well.

Let $V_{i}^{1}=\left(v_{1}^{1}, \ldots, v_{c_{i}}^{1}\right)$ be the restriction of $V_{i}$ to those indices $j$ with $b_{j}^{\prime}=1$. Let $V_{i}^{0}=\left(v_{1}^{0}, \ldots, v_{d_{i}}^{0}\right)$ be the restriction of $V_{i}$ to those indices $j$ with $b_{j}^{\prime}=0$. Finally, we put $A_{i}=\left(f\left(v_{1}^{1}\right), \ldots, f\left(v_{c_{i}}^{1}\right)\right)$ and $P_{i}=\left(p_{1}, \ldots, p_{d_{i}}\right)$ where $p_{j}$ is chosen so $f\left(v_{j}^{0}\right)=f\left(v_{p_{j}}^{1}\right)$.

Initially $\left(B^{\prime}, A, P\right)$ consists of three empty lists, and whenever it comes to uncoloring a violator $H_{i}$ in the course of the algorithm then $B_{i}^{\prime}$ is appended to $B^{\prime}, A_{i}$ is appended to $A$, and $P_{i}$ is appended to $P$. Finally, we merge $A$ and $P$ into a single list ${ }^{*} A P$ by reversing $A$ and appending $P$ (only for technical reasons to simplify the counting). This will yield $\Gamma=\left(B^{\prime},{ }^{*} A P\right)$.

The last component $F$ of $\operatorname{LOG}(X)$ consists of the partial coloring $f$ of $G$ when the algorithm stops, i.e., after the $M$ iterations of the loop. More explicitly, $F=\left(f\left(v_{1}\right), f\left(v_{2}\right), \ldots, f\left(v_{n}\right)\right)$ where the coloring $f$ is taken from the output.

For the counting it will be convenient to have lists of length independent of $X$. Let $\gamma$ be the number of colored vertices in $F$, i.e., the number of $v_{i}$ with $f\left(v_{i}\right) \neq \vartheta$. Note that $M-\sum m_{i}=\gamma$. We append $\gamma$ entries to the lists $B^{\prime},{ }^{*} A P$ from $\Gamma$ and $2 \gamma+2 v$ entries to $B$ from $\Sigma$, where $v$ is the total number of violators. Now $|B|=2 M,\left|B^{\prime}\right|=M$, and $|* A P|=M$. The length of $L$ is $\sum\left(m_{i}-1\right) \leq(M-\gamma)\left(1-\frac{1}{2 p}\right) \leq\left(1-\frac{1}{2 p}\right) M$ and we append elements to $L$ to make the length of $L$ equal to this value.

Claim. The mapping $X \rightarrow \operatorname{LOG}(X)$ is injective.

Proof. We will show how to reconstruct the original input $X$ from the value of $\log (X)$. The reconstruction is done in two phases. In the forward phase we reconstruct:

(i) $\left(w_{1}, w_{2}, \ldots, w_{M}\right)$ where $w_{i}$ is the vertex colored with $x_{i}$ at line 6 in the $i$-th iteration;

(ii) $\left(U_{0}, U_{1}, \ldots, U_{M}\right)$ where $U_{i}$ is the set of uncolored vertices after the $i$-th iteration.

(iii) $W_{i}^{\prime}$ for every $i$ such that the algorithm encounters a violator in the $i$-th step, where $W_{i}^{\prime}$ is the traversal of the subgraph of $H_{i}$ with all uncolored vertices, fixed in the definition of $\operatorname{LOG}(X)$. 
Note first that $U_{0}=V(G)$. For the two lists in $\Sigma \in \operatorname{LOG}(X)$ we initialize $\operatorname{pos}(B)=0$ and $\operatorname{pos}(L)=0$. The invariant is that when $U_{i-1}$ has been computed $\operatorname{pos}(B)$ and $\operatorname{pos}(L)$ mark the tail of the lists constructed up to the end of the $(i-1)$-st iteration of the loop.

Let $i \geq 1$ and suppose that $U_{i-1}$ is determined. Then the vertex $w_{i}$ is identified as the first element of $U_{i-1}$ in $\Pi$. Now, if $z_{i}=z_{i-1}+1$ then there was no violator in the $i$-th iteration step and we simply have $U_{i}=U_{i-1}-\left\{w_{i}\right\}$.

If $z_{i} \leq z_{i-1}$, then the coloring of $w_{i}$ led to a violator $H_{i}$ whose vertices were partially uncolored. The number of uncolored vertices of $H_{i}$ can be identified as $m_{i}=z_{i-1}+1-z_{i}$. Knowing $m_{i}$ we can separate the blocks of the lists $B$ and $L$ corresponding to the subgraph $H_{i}$. Let $B_{i}=\left(b_{0}, b_{1}, b_{2}, \ldots, b_{2 m_{i}-3}\right)$ be the block of $2 m_{i}-2$ entries of $B$ starting from the current position $\operatorname{pos}(B)$ and advance $\operatorname{pos}(B) \leftarrow \operatorname{pos}(B)+2 m_{i}-2$. Let $L_{i}=\left(\ell_{1}, \ldots, \ell_{m_{i}-1}\right)$ be the block of $m_{i}-1$ entries of $L$ starting from the current position $\operatorname{pos}(L)$ and advance $\operatorname{pos}(L) \leftarrow \operatorname{pos}(L)+m_{i}-1$. Having identified $w_{i}, B_{i}$, and $L_{i}$ we can reconstruct the traversal $W_{i}^{\prime}=\left(u_{0}^{\prime}, u_{1}^{\prime}, u_{2}^{\prime}, \ldots, u_{2 m_{i}-2}^{\prime}\right)$ that was fixed computing the value of $\operatorname{LOG}(X)$. Indeed, $u_{0}^{\prime}=w_{i}$. We keep track of the number $k$ of the next forward step to come, so we start with $k=1$. When we have identified the vertex $u_{j}^{\prime}$, for $j \geq 0$, then the value $b_{j}$ indicates whether the next step is forward or backward. If the step is forward, then we know that the $u_{j}^{\prime} u_{j+1}^{\prime}$ edge is the $\ell_{k}$-th edge in the fixed ordering of edges adjacent to $u_{j}^{\prime}$. Therefore, we identified $u_{j+1}^{\prime}$ and we increment $k$. If the step is backward, then we identify $u_{j+1}^{\prime}$ from the discovered so far subtree of $T_{i}$. When the sequence $W_{i}^{\prime}=\left(u_{0}^{\prime}, u_{1}^{\prime}, u_{2}^{\prime}, \ldots, u_{2 m_{i}-2}^{\prime}\right)$ is reconstructed, in particular, we have identified all the uncolored vertices of $H_{i}$. Finally, $U_{i}=U_{i-1} \cup\left\{u_{0}^{\prime}, u_{1}^{\prime}, u_{2}^{\prime}, \ldots, u_{2 m_{i}-2}^{\prime}\right\}$. This completes the description of the forward phase.

In the backward phase we reconstruct:

(i) $\left(f_{M}, f_{M-1}, \ldots, f_{1}\right)$ where $f_{i}$ is the partial coloring $f$ after the $i$-th iteration; and

(ii) $\left(x_{M}, x_{M-1}, \ldots, x_{1}\right)$.

Note that $f_{M}=F$ is a part of the evaluation of $\operatorname{LOG}(X)$. In particular, the number $\gamma$ of vertices colored after the whole process is known. For the two lists in $\Gamma \in \operatorname{LOG}(X)$ we initialize $\operatorname{pos}\left(B^{\prime}\right)=M-\gamma$, $\operatorname{pos}(A)=0$, and $\operatorname{pos}(P)=M-\gamma$. The invariant is that when $f_{i}$ has been computed these functions mark the tail of the lists after the $(i-1)$-st iteration of the loop. In order to mark the tail of list $A$, we mark the head of the respective part of ${ }^{*} P$, because $A$ is reversed there. The tail of $P$ and ${ }^{*} A P$ coincide except for the $\gamma$ added elements.

Let $1<i \leq M$ and suppose that $f_{i}$ has been identified. Now, if $z_{i}=z_{i-1}+1$ then there was no violator in the $i$-th iteration step and we simply have $x_{i}=f_{i}\left(w_{i}\right)$ and $f_{i-1}$ is obtained from $f_{i}$ by uncoloring $w_{i}$.

If $z_{i} \leq z_{i-1}$, then the coloring of $w_{i}$ led to a violator $H_{i}$. From the forward phase we know the traversal $W_{i}^{\prime}=\left(u_{0}^{\prime}, u_{1}^{\prime}, u_{2}^{\prime}, \ldots, u_{2 m_{i}-2}^{\prime}\right)$ with $u_{0}^{\prime}=w_{i}$ of a spanning tree of the subgraph of $H_{i}$ uncolored in the $i$ th step. Let $V_{i}=\left(v_{1}^{\prime}, u_{2}^{\prime}, \ldots, v_{m_{i}}^{\prime}\right)$ be the uncolored vertices of $H_{i}$ sorted by their first-appearance in $W_{i}^{\prime}$. From $B^{\prime}$ take the block $B_{i}^{\prime}=\left(b_{1}^{\prime}, \ldots, b_{m_{i}}^{\prime}\right)$ of length $m_{i}$ preceding the current position. The sequence $B_{i}^{\prime}$ guides us how to identify the split of $V_{i}$ into $V_{i}^{1}=\left(v_{1}^{1}, \ldots, v_{c_{i}}^{1}\right)$ and $V_{i}^{0}=\left(v_{1}^{0}, \ldots, v_{d_{i}}^{0}\right)$. They are simply the restrictions of $V_{i}$ to those indices $j$ with $b_{j}^{\prime}=1$ and $b_{j}^{\prime}=0$, respectively. As we see above, from the lengths of the identified sequences we can reconstruct the values of $c_{i}$ and $d_{i}$, i.e., $c_{i}=\left|V_{i}^{1}\right|$ and $d_{i}=\left|V_{i}^{0}\right|$. Now we can identify the next block $A_{i}=\left(\alpha_{1}, \ldots, \alpha_{c_{i}}\right)$ of length $c_{i}$ in ${ }^{*} P$ by reversing the next $c_{i}$ elements after $\operatorname{pos}(A)$ and the block $P_{i}=\left(p_{1}, \ldots, p_{d_{i}}\right)$ of size $d_{i}$ preceding the position $\operatorname{pos}(P)$ in ${ }^{*} A P$. The list $A_{i}$ contains the colors of vertices in $V_{i}^{1}$, namely, $f_{i-1}\left(v_{j}^{1}\right)=\alpha_{j}$. For vertices in $V_{i}^{0}$ we use the list $P_{i}$ and have $f_{i-1}\left(v_{j}^{0}\right)=\alpha_{p_{j}}$. Hence, we finally get $x_{i}=\alpha_{1}$ and after resetting $f_{i-1}\left(w_{i}\right)=\diamond$ we have 


\section{IMPROVED BOUNDS FOR CENTERED COLORINGS AUTHOR}

reconstructed the coloring $f_{i-1}$. It only remains to update $\operatorname{pos}\left(B^{\prime}\right) \leftarrow \operatorname{pos}\left(B^{\prime}\right)-m_{i}, \operatorname{pos}(A) \leftarrow \operatorname{pos}(A)+c_{i}$, and $\operatorname{pos}(P) \leftarrow \operatorname{pos}(P)-d_{i}$. This completes the description of the backward phase and the proof of the claim.

We now come to the final task in the proof, we estimate the size of the image of the LoG-function. Recall that $\operatorname{LOG}(X)=(Z, \Sigma, \Gamma, F)$, we will independently estimate the number of values each of the four components can take.

Recall that $Z=\left(z_{0}, z_{1}, \ldots, z_{M}\right)$. Based on $Z$, we build an auxiliary sequence $S_{Z}=\left(s_{1}, \ldots, s_{2 M}\right)$ where each $s_{i}$ is either +1 or -1 . The sequence is obtained from left to right. Set $s_{1}=z_{1}-z_{0}=1$. Assume that $z_{0}, \ldots z_{j}$ have been processed. If $z_{j+1}-z_{j}=1$, then append +1 to $S_{Z}$ and if $z_{j+1}-z_{j}=-r \leq 0$ append a single +1 and a sequence of $r+1$ entries of -1 to $S_{Z}$, finally add a sequence of $\gamma$ entries of -1 . Note that replacing $Z$ by $S_{Z}$ in $\operatorname{LOG}(X)$ would not change the encoded information. Indeed knowing $\gamma$ from $F \in \operatorname{LOG}(X)$ the final sequence can be removed and $z_{i}$ is obtained as the sum of the $i$-th +1 entry and the block of -1 entries following it in the truncated $S_{Z}$.

In $S_{Z}$ the number of +1 entries and the number of -1 entries are exactly $M$ and every prefix of $S_{Z}$ has a non-negative sum. These sequences are known as Dyck-paths and counted by the Catalan number $C_{M}$. It is well-known that $C_{M}$ is in $o\left(4^{M}\right)$.

For $\Sigma$ and $\Gamma$ we consider the data accumulated with a violator $H_{i}$ and its $m_{i}$ uncolored vertices. In $\Sigma=(B, L)$ we add a binary list $B_{i}$ of length $2 m_{i}-2$ and a list $L_{i}$ of length $m_{i}-1$ with entries in $\{1, \ldots, \Delta\}$. In $\Gamma=\left(B^{\prime},{ }^{*} A P\right)$ we add a binary list $B_{i}^{\prime}$ of length $m_{i}$, a list $A_{i}$ of length $c_{i}$ with entries in $\{1, \ldots, c\}$, and a list $P_{i}$ of length $d_{i}$ with entries in $\{1, \ldots, p\}$. From the properties of a violator we have $c_{i}+d_{i}=m_{i}$ and $d_{i} \geq c_{i}$.

Since we know the lengths of these lists, we can upper bound the possibilities for $B, L$, and $B^{\prime}$ respectively by $2^{2 M}, \Delta^{\left(1-\frac{1}{2 p}\right) M}$, and $2^{M}$. Lists $A$ and $P$ together have $M$ entries and for each $i$ we have $d_{i} \geq c_{i}$. Since $c>p$ we only overcount when assuming that $A$ and $P$ are of the same length, therefore, the number of possibilities for ${ }^{*} A P$ is bounded by $c^{M / 2} p^{M / 2}$.

For the final coloring $F$ there are $(c+1)^{n}$ options.

This way we obtain an upper bound on the size of the image of the LOG-function

$$
o\left(4^{M}\right) \cdot 2^{2 M} \Delta^{\left(1-\frac{1}{2 p}\right) M} 2^{M} c^{M / 2} p^{M / 2}(c+1)^{n} .
$$

Since LOG is injective we get

$$
\begin{gathered}
o\left(4^{M}\right) \cdot 2^{2 M} \Delta^{\left(1-\frac{1}{2 p}\right) M} 2^{M} c^{M / 2} p^{M / 2} \cdot(c+1)^{n} \geq c^{M}, \\
o\left(32^{M}\right) \cdot \Delta^{\left(1-\frac{1}{2 p}\right) M} p^{M / 2} \cdot(c+1)^{n} \geq c^{M / 2}, \\
o\left(\left(2^{10} \cdot \Delta^{2-1 / p} \cdot p\right)^{M}\right) \cdot(c+1)^{2 n} \geq c^{M} .
\end{gathered}
$$

Since we have chosen $c=\left\lceil 2^{10} \Delta^{2-1 / p} p\right\rceil$, we see that for large enough $M$ the inequality above cannot hold. Therefore, for some input $X$ the algorithm outputs a $p$-centered coloring of $G$ using $c$ colors. This completes the proof.

Let $c=\left\lceil 2^{10} \Delta^{2-1 / p} p\right\rceil$. If the naïve randomized coloring algorithm is allowed to use $2 c$ colors it succeeds in finding a valid coloring in expected $\leq 2 n \log (2 c+1)+4$ iterations. Indeed, let $M=$ 
$2 n \log (2 c+1)$ and consider the probability that after $M+t$ iterations the algorithm did not break. This probability is upper bounded by $\frac{c^{(M+t) / 2}(2 c+1)^{n}}{(2 c)^{(M+t) / 2}} \leq \frac{1}{2^{t / 2}}$, whence the expected number of iterations is upper bounded by $M+\sum \frac{1}{2^{t / 2}}<M+4$.

A single iteration step can be done in $\mathcal{O}(n)$ time. Indeed, the most time consuming thing to do is to check whether after coloring $w_{i}$ there is a violator. This can be done by iterating over all subsets $C \subset\{1, \ldots, c\}$ of size at most $p$. For each such $C$, we can determine in $\mathcal{O}(n)$ time if the component of the subgraph spanned by vertices with colors from $C$ that contains $w_{i}$ is a violator. Since the number of such subsets is a function of $c$ and $p$ only, i.e., of $\Delta$ and $p$, we conclude that a single iteration can be done in $\mathcal{O}(n)$ time.

Therefore, the expected runtime of the randomized algorithm is $\mathcal{O}\left(n^{2}\right)$.

\section{Upper bound for graphs excluding a fixed topological minor: Lifting through the structure theorem}

In this section we prove Theorem 4. We start with an informal statement of the structure theorem by Grohe and Marx [7]. For every graph $H$, every graph excluding $H$ as a topological minor has a tree-decomposition with bounded adhesion such that every torso either has bounded degree with the exception of a bounded number of vertices, or excludes a fixed graph as a minor. Furthermore, such a decomposition for a graph $G$ can be computed in time $f(H) \cdot|V(G)|^{\mathcal{O}(1)}$ for some computable function $f$.

We proceed with all the necessary definitions.

A graph $H$ is a minor of a graph $G$ if $H$ can be obtained from $G$ by deleting vertices, deleting edges, and contracting edges. A graph $H$ is a topological minor of a graph $G$ if $H$ can be obtained from $G$ by deleting vertices, deleting edges, and contracting edges with at least one endpoint of degree 2.

A tree-decomposition of a graph $G$ is a pair $\left(T,\left\{B_{t}\right\}_{t \in V(T)}\right)$ where $T$ is a tree and the sets $B_{t}(t \in V(T))$ are subsets of $V(G)$ called bags satisfying

1. for each edge $u v \in E(G)$ there is a bag containing both $u$ and $v$, and

2. for each vertex $v \in V(G)$ the set of vertices $t \in V(T)$ with $v \in B_{t}$ induces a non-empty subtree of $T$. The treewidth of a graph $G$ is the minimum $k$ such that $G$ has a tree-decomposition with all bags of size at most $k+1$. An adhesion set in the tree-decomposition $\left(T,\left\{B_{t}\right\}_{t \in V(T)}\right)$ of $G$ is a set $B_{t} \cap B_{t^{\prime}}$ where $t t^{\prime} \in E(T)$. The adhesion of a tree-decomposition is the maximum size of an adhesion set. The torso of a bag $B_{t}$ is the graph obtained from $G\left[B_{t}\right]$ by adding all the edges between vertices in every adhesion set $B_{t} \cap B_{t^{\prime}}$ where $t t^{\prime} \in E(T)$.

We are ready to present the precise statement of the structure theorem for graphs avoiding a fixed graph as a topological minor.

Theorem 11 ([7]). For every integer $k \geq 1$, there exist $a(k), c(k), d(k)$, and $e(k)$ such that the following holds: If $H$ is a graph on $k$ vertices and $G$ avoids $H$ as a topological minor, then $G$ has a tree-decomposition $\left(T,\left\{B_{t}\right\}_{t \in V(T)}\right)$ with adhesion at most a $(k)$ such that for every $t \in V(T)$ :

(i) the torso of $B_{t}$ has at most $c(k)$ vertices of degree larger than $d(k)$, or

(ii) the torso of $B_{t}$ avoids $K_{e(k)}$ as a minor. 


\section{IMPROVED BOUNDS FOR CENTERED COLORINGS AUTHOR}

To handle tree-decompositions of bounded adhesion we use the following lemma from [21]. We say that a tree-decomposition $\left(T,\left\{B_{t}\right\}_{t \in V(T)}\right)$ of a graph $G$ is over a class of graphs $\mathcal{C}$ if for every $t \in V(T)$ the torso of $B_{t}$ lies in $\mathrm{C}$.

Lemma 12 ([21]). Let $\mathcal{C}$ be a class of graphs such that for a fixed $d$ and every $p \geq 1$, every graph in $\mathcal{C}$ admits a p-centered coloring with $\mathcal{O}\left(p^{d}\right)$ colors. If $\mathcal{D}$ is a class of graphs such that every graph $G \in \mathcal{D}$ has a tree-decomposition over $\mathcal{C}$ with adhesion at most $k$, then for every $p \geq 1$, every graph in $\mathcal{D}$ admits a p-centered coloring with $\mathcal{O}\left(p^{d+k}\right)$ colors.

We also need the following theorem from [21].

Theorem 13 ([21]). For every graph $H$ there is a polynomial $f$ such that the graphs excluding $H$ as a minor admit p-centered colorings with at most $f(p)$ colors.

With these tools in hand we can give a proof of a polynomial upper bound for graphs avoiding a fixed topological minor (Theorem 4).

Let $H$ be a graph on $k$ vertices and let $G$ be a graph avoiding $H$ as a topological minor. From Theorem 11 we obtain a tree-decomposition $\left(T,\left\{B_{t}\right\}_{t \in V(T)}\right)$ with adhesion at most $a(k)$ such that every torso of a bag in the tree-decomposition satisfies one of the two properties mentioned there.

For fixed $p \geq 1$, we construct a $p$-centered coloring of $G$. Let $t \in V(T)$ and consider the torso $\tau(t)$ of the bag $B_{t}$. Suppose that $\tau(t)$ has at most $c(k)$ vertices of degree larger than $d(k)$. We color the vertices of $\tau(t)$ as follows:

1. use at most $c(k)$ distinct colors on vertices of degree larger than $d(k)$;

2. color the remaining vertices using at most $(32 \cdot d(k))^{2} \cdot p$ colors applying Theorem 3 .

Clearly, we obtain a $p$-centered coloring of $\tau(t)$ using at most $c(k)+(32 \cdot d(k))^{2} \cdot p$ colors.

Now suppose that $\tau(t)$ avoids $K_{e(k)}$ as a minor. By Theorem 13, there is a constant $f(k)$ such that $\tau(t)$ admits a $p$-centered coloring using $\mathcal{O}\left(p^{f(k)}\right)$ colors.

Therefore, by Lemma 12, the graph $G$ admits a $p$-centered coloring using $\mathcal{O}\left(p^{1+f(k)+a(k)}\right)$ colors. This completes the proof.

As mentioned, Theorem 11 comes with an algorithm that produces a desired tree-decomposition for a graph $G$ on $n$ vertices in $f(H) \cdot n^{\mathcal{O}(1)}$ in time for some computable function $f$. Indeed, both results from [21], Lemma 12 and Theorem 13, are also supported by algorithms with running times $n^{\mathcal{O}(1)}$. The final algorithm within the proof of our theorem calls the algorithm for bounded degree case given by Theorem 3. As we have seen in the previous section, the expected running time of the latter algorithm is $\mathcal{O}\left(n^{2}\right)$. All in all, there is a randomized polynomial time algorithm to determine a $p$-centered coloring of a graph without $H$ as a topological minor with polynomial in $p$ number of colors.

\section{Upper bounds for graphs of bounded simple treewidth}

Theorem 1 has been shown as a part of Corollary 10. There we made use of an upper bound for planar graphs of treewidth at most 3 (Theorem 6.(iii)). We remark that planar graphs of treewidth at most 3 are also known as subgraphs of stacked triangulations, see [11]. We proceed to prove Theorem 6.(i) and 
Theorem 7.(i) which also gives Theorem 6.(iii) To establish these results, we need a structural property of chordal graphs, sometimes called the shadow completeness, that was independently observed by Dujmović, Morin and Wood [4] and by Kündgen and Pelsmajer [13].

Lemma 14 (shadow completeness). Let $G$ be a chordal graph and let $\left(V_{0}, V_{1}, \ldots\right)$ be a BFS-layering of $G$. Let $i>0$ and let $C \subseteq G\left[\bigcup_{j>i} V_{j}\right]$ be connected. Let the shadow $S_{i}(C)$ of $C$ in $V_{i}$ be the set of $v \in V_{i}$ such that there is a path $P$ from $v$ to $C$ with $P \cap V_{i}=\{v\}$. Then $S_{i}(C)$ is a clique. Moreover, $S_{i}(C)$ separates $C$ from $G\left[\bigcup_{j<i} V_{j}\right]$.

Proof. Let $C$ be a connected subgraph of $G\left[\bigcup_{j>i} V_{j}\right]$. Let $v$ and $v^{\prime}$ be two vertices in $S_{i}(C)$. Let $P$ and $P^{\prime}$ be the certifying paths of $v$ and $v^{\prime}$. The ends of $P$ and $P^{\prime}$ can be connected in $C$ yielding a path connecting $v$ and $v^{\prime}$ which has all inner vertices in $G\left[\bigcup_{j>i} V_{j}\right]$. Let $P_{1}$ be a shortest path from $v$ to $v^{\prime}$ with all inner vertices in $G\left[\bigcup_{j>i} V_{j}\right]$. Let $P_{2}$ be a shortest path from $v$ to $v^{\prime}$ with all inner vertices in $\bigcup_{j<i} V_{j}$ (there is one as $G\left[\bigcup_{j<i} V_{j}\right]$ is connected in the BFS-tree). Now $P_{1} \cup P_{2}$ is a cycle in $G$ of length at least 4 and the only possible chord is an edge $v v^{\prime}$. Since $G$ is chordal, we have $v v^{\prime} \in E(G)$, as desired.

Since every path from a vertex in $\bigcup_{j<i} V_{j}$ to a vertex in $C$ contains a vertex in $V_{i}$ the shadow $S_{i}(C)$ is a separator for these two sets.

Corollary 15. Let $G$ be a chordal graph and let $\left(V_{0}, V_{1}, \ldots\right)$ be a BFS-layering of $G$. Let $i \geq 0$ and let $H \subseteq G\left[\bigcup_{j \geq i} V_{j}\right]$ be a connected induced subgraph. Then $H \cap G\left[V_{i}\right]$ is connected.

Proof. Consider two vertices $u, v$ in $H \cap G\left[V_{i}\right]$. Take a path $P$ from $u$ to $v$ in $H$. If all vertices of $P$ are in $H \cap G\left[V_{i}\right]$, then we are done. Otherwise, consider a vertex $x$ of $P$ with $x \in V_{i}$ such that the vertex after $x$ in $P$ is not in $V_{i}$. Let $y$ be the first vertex of $P$ after $x$ with $y \in V_{i}$. Lemma 14 implies that $x$ and $y$ are adjacent. Thus $P$ is not a shortest path between $u$ and $v$ in $H$, a contradiction.

\subsection{Proof of the upper bound for outerplanar graphs}

Let $G$ be an outerplanar graph. Then $G$ is a subgraph of a maximal outerplanar graph $G^{+}$. Since any $p$-centered coloring of $G^{+}$can be restricted to $G$, we may assume $G=G^{+}$. It is well-known that maximal outerplanar graphs are 2-trees. In particular, $G$ is chordal. Let $\left(V_{0}, V_{1}, \ldots\right)$ be a BFS-layering of $G$.

Claim. The subgraph $G\left[V_{i}\right]$ of $G$ induced by $V_{i}$ is a linear forest, i.e., each connected component of it is a path.

Proof. Contracting $G\left[\bigcup_{j<i} V_{j}\right]$ and deleting $G\left[\bigcup_{j>i} V_{j}\right]$ yields a minor $M$ of $G$ that consists of $G\left[V_{i}\right]$ and an additional apex $a$ adjacent to every $v \in V_{i}$. Since adding an apex increases the treewidth by exactly one, we have $\operatorname{tw}\left(G\left[V_{i}\right]\right) \leq 1$, so $G\left[V_{i}\right]$ is a forest. Assume $v \in G\left[V_{i}\right]$ has (at least) 3 neighbors $x, y, z \in G\left[V_{i}\right]$. Deleting the edge $a v$ and all vertices except $x, y, z, v, a$ from $M$ yields a $K_{2,3}$-minor of $G$, which is a contradiction with $G$ being outerplanar. Thus $G\left[V_{i}\right]$ is a forest with all vertices of degree at most 2, as desired.

We will refer to the connected components of $G\left[V_{i}\right]$ as the layer paths of $G\left[V_{i}\right]$. In the following, we construct a coloring $\phi: V(G) \rightarrow C$ with $C$ being a set of colors of size $p\lceil\log (p+1)\rceil+2 p+1$. 


\section{IMPROVED BOUNDS FOR CENTERED COLORINGS AUTHOR}

We construct $\phi$ layer by layer. In each layer we color the layer paths independently. When it comes to coloring a layer path $P$ we have a set $\mathcal{F}(P)$ of colors which are forbidden for $P$. We initialize $\mathcal{F}(P)=\emptyset$ for every layer path $P$ in $G$. The set $\mathcal{F}(P)$ will be of size at most $p\lceil\log (p+1)\rceil+p$. The path $P$ will be colored with a set of $p+1$ colors from $C-\mathcal{F}(P)$.

The layer $V_{0}$ contains only a single vertex $v$. Set $\phi(v)=\alpha$ for some color $\alpha \in C$. To make sure that $\alpha$ does not appear on the next $p$ layers we add $\alpha$ into $\mathcal{F}(P)$ for every layer path $P$ of $G\left[V_{i}\right]$ with $0<i \leq p$.

If all layers $V_{j}$ with $0 \leq j<i$ are colored and $P$ is a layer path of $G\left[V_{i}\right]$, then we extend $\phi$ to $P$ as follows: Choose a set $C_{P} \subset C-\mathcal{F}(P)$ of $p+1$ colors, say $C_{P}=\left\{0_{P}, 1_{P}, \ldots, p_{P}\right\}$. Color $P=\left(v_{0}, v_{1}, \ldots, v_{m}\right)$ periodically, i.e., let $\phi\left(v_{k}\right)=j_{P}$ if $k=j \bmod (p+1)$.

Now consider a layer path $P^{\prime}$ in $G\left[V_{j}\right]$ with $i<j$, and note that the shadow $S_{i}\left(P^{\prime}\right)$ of $P^{\prime}$ in $V_{i}$ is the set

$$
\left\{v \in V_{i} \mid v \text { is adjacent to the component of } G\left[\bigcup_{j^{\prime}>i} V_{j^{\prime}}\right] \text { containing } P^{\prime}\right\} \text {. }
$$

By Lemma 14 the set $S_{i}\left(P^{\prime}\right)$ induces a clique in $G\left[V_{i}\right]$. Therefore, $S_{i}\left(P^{\prime}\right)$ is contained in one of the layer paths of $G\left[V_{i}\right]$ and $\left|S_{i}\left(P^{\prime}\right)\right| \leq 2$. Moreover $S_{i}\left(P^{\prime}\right)$ is a separator. For $i<k<j$ let $P_{k}$ be the layer path of $V_{k}$ containing $S_{k}\left(P^{\prime}\right)$. The separator property implies the following transitivity of shadows: $S_{i}\left(P^{\prime}\right)=S_{i}\left(P_{k}\right)$. Indeed, a path from $V_{i}$ to $P^{\prime}$ has to pass through $S_{k}\left(P^{\prime}\right) \subset P_{k}$ and every path from $V_{i}$ to $P_{k}$ can be extended within $G\left[\bigcup_{j^{\prime} \geq k} V_{j^{\prime}}\right]$ to a path from $V_{i}$ to $P^{\prime}$.

For each layer path $P^{\prime}$ in $G\left[V_{k}\right]$ with $i<k \leq i+p$ such that $S_{i}\left(P^{\prime}\right) \subseteq V(P)$ we extend $\mathcal{F}\left(P^{\prime}\right)$ by some colors used on $P$. To determine these forbidden colors we use an auxiliary structure. Let $T$ be a binary tree on $p$ nodes with root $r_{T}$ and height $\lceil\log (p+1)\rceil$ where height of a rooted tree is the maximum number of nodes on a path from the root to a leaf. We label the nodes of $T$ with the totally ordered set of colors $1_{P}<2_{P} \ldots<p_{P}$ using an in-order traversal, i.e., the label of each node is larger than all labels of nodes in its left subtree and smaller than all labels of nodes in its right subtree. For $\ell \in\left\{1_{P}, \ldots, p_{P}\right\}$ let $F(\ell)$ denote the set of labels of ancestors of the node $u$ labeled $\ell$, i.e., $F(\ell)$ is the set of labels seen on the path from $u$ to $r_{T}$ in $T$. We extend this definition by $F\left(0_{P}\right)=\emptyset$.

Let $i<j \leq i+p$ and consider a layer path $P^{\prime}$ of $G\left[V_{j}\right]$ with $S_{i}\left(P^{\prime}\right) \subset V(P)$. We extend the set of forbidden colors of $P^{\prime}$ putting

$$
\mathcal{F}\left(P^{\prime}\right) \leftarrow \mathcal{F}\left(P^{\prime}\right) \cup\left\{0_{P}\right\} \cup \bigcup_{v \in S_{i}\left(P^{\prime}\right)} F(\phi(v)) .
$$

Claim. If $\ell, \ell^{\prime}$ are consecutive in the cyclic order on $\left\{0_{P}, 1_{P}, \ldots, p_{P}\right\}$, then

$$
F(\ell) \subseteq F\left(\ell^{\prime}\right) \text { or } F\left(\ell^{\prime}\right) \subseteq F(\ell) .
$$

Proof. If either $\ell$ or $\ell^{\prime}$ is 0 , the statement is obvious. Otherwise $\ell^{\prime}=\ell+1$ and there are nodes $u$ and $u^{\prime}$ with these labels in $T$. It is easy to see that every two consecutive nodes in an in-order traversal must be in the ancestor-descendant relation in the tree. This immediately gives $F(\ell) \subseteq F\left(\ell^{\prime}\right)$ or $F\left(\ell^{\prime}\right) \subseteq F(\ell)$.

Note that if $S_{i}\left(P^{\prime}\right)=\left\{v, v^{\prime}\right\}$, then $v$ and $v^{\prime}$ are consecutive on $P$. By the claim proven above we have

$$
\left|\bigcup_{v \in S_{i}\left(P^{\prime}\right)} F(\phi(v))\right| \leq\lceil\log (p+1)\rceil .
$$

Hence at most $\lceil\log (p+1)\rceil+1$ colors are added to $\mathcal{F}\left(P^{\prime}\right)$ after coloring the layer paths in $G\left[V_{i}\right]$. Since $\mathcal{F}\left(P^{\prime}\right)$ is extended at most $p$ times we get an upper bound

$$
\left|\mathcal{F}\left(P^{\prime}\right)\right| \leq p\lceil\log (p+1)\rceil+p .
$$


Claim. $\phi$ is a p-centered coloring of $G$.

Proof. Let $H$ be a connected subgraph of $G$. We want to show that $\phi$ uses more than $p$ colors on $H$ or there is a color used only once on $H$. Let $i:=\min \left\{j \geq 0 \mid V_{j} \cap V(H) \neq \emptyset\right\}$. We distinguish two cases:

Case 1. $H$ contains a vertex from $V_{i+p}$.

Let $Q$ be a layer path in $V_{i+p}$ containing a vertex $u_{i+p}$ from $H$. For all $j$ with $i \leq j<i+p$ the connectivity of $H$ together with the separator property of $S_{j}(Q)$ imply that $S_{j}(Q) \cap V(H) \neq \emptyset$. Therefore we can choose $u_{j} \in S_{j}(Q) \cap V(H)$ for $i \leq j<i+p$.

We claim that $\phi\left(u_{j}\right) \neq \phi\left(u_{k}\right)$ for all $i \leq j<k \leq i+p$. Let $Q_{k}$ be the layer path containing $u_{k}$. From $S_{k}(Q) \subset Q_{k}$ it follows from the transitivity property of shadows that $S_{j}\left(Q_{k}\right)=S_{j}(Q)$, whence $u_{j} \in S_{j}\left(Q_{k}\right)$. Now $\phi\left(u_{j}\right) \in F\left(\phi\left(u_{j}\right)\right) \subset \mathcal{F}\left(Q_{k}\right)$, Therefore, the color $\phi\left(u_{j}\right)$ is forbidden for the path $Q_{k}$ and $\phi\left(u_{k}\right) \neq \phi\left(u_{j}\right)$.

We conclude that the vertices $u_{i}, \ldots, u_{i+p}$ are colored with $p+1$ distinct colors. Therefore $\phi$ uses more than $p$ colors on $H$.

Case 2. $H$ contains no vertex from $V_{i+p}$.

Corollary 15 implies that $Q=G\left[V(H) \cap V_{i}\right]$ is connected, so it is a subpath of some layer path $P$ of $G\left[V_{i}\right]$. Let $C_{P}=\left\{0_{P}, \ldots, p_{P}\right\}$ be the set of colors used to color $P$. Let $C_{Q} \subset C_{P}$ be the set of colors used on $Q$. If two vertices of $Q$ have the same color, then due to the periodicity of $\phi$ on $P$ all the $p+1$ colors from $\left\{0_{P}, \ldots, p_{P}\right\}$ are used on $Q$, whence $\phi$ uses on $H$ more than $p$ colors. From now on we assume that all colors on $Q$ are unique.

If $0_{P} \in C_{Q}$, then recall that $0_{P} \in \mathcal{F}\left(P^{\prime}\right)$ for any layer path $P^{\prime}$ of $G\left[V_{k}\right]$ with $i<k \leq i+p$ such that $S_{i}\left(P^{\prime}\right) \subseteq V(P)$. Therefore $0_{P}$ is contained in $\mathcal{F}\left(P^{\prime}\right)$ for all $P^{\prime}$ intersected by $V(H)$. Hence $0_{P}$ is a unique color on $H$. From now on we assume that $0_{P} \notin C_{Q}$.

Consider the rooted binary tree $T$ on $p$ nodes devised during the coloring procedure of $P$. Since $C_{Q}$ is an interval of $\{1, \ldots, p\}$ there is a unique node $r_{Q}$ in $T$ with a label from $C_{Q}$ such that $r_{Q}$ is a predecessor of all nodes with labels from $C_{Q}$ in $T$. Let $\beta$ be the label of $r_{Q}$. It follows that $\beta \in F(\ell)$ for all $\ell \in C_{Q}$, whence $\beta \in F(\phi(v))$ for all $v \in Q$. Therefore $\beta \in \mathcal{F}\left(P^{\prime}\right)$ for any layer path $P^{\prime}$ of $G\left[V_{k}\right]$ with $i<k \leq i+p$ such that $S_{i}\left(P^{\prime}\right) \subseteq V(P)$. This means that $\beta$ is unique on $H$, as desired.

In summary, any outerplanar graph has a $p$-centered coloring with $p\lceil\log (p+1)\rceil+2 p+1 \epsilon$ $\mathcal{O}(p \log p)$. This completes the proof of Theorem 6.(i). The described algorithm coloring outerplanar graphs has a straightforward $\mathcal{O}(n)$ time implementation.

\subsection{Proof of the upper bound for graphs of bounded simple treewidth}

For $p \geq 1$, let $f(p)$ be the maximum $\chi_{p}(G)$ when $G$ is outerplanar. We have just seen that $f(p) \in$ $\mathcal{O}(p \log p)$. Now we are going to show that every planar graph of treewidth at most 3 admits a $p$-centered coloring with at most $(p+1) f(p)$ colors. This proof can be generalized though, which is what we will do here. The essence of the generalization is that maximal outerplanar graphs are simple 2-trees. We continue with a discussion of simple treewidth.

For $k \geq 1$, a tree-decomposition $(T, \mathcal{B})$ of $G$ is $k$-simple if $(T, \mathcal{B})$ is of width at most $k$ and for every $X \subset V(G)$ with $|X|=k$, we have that $X \subset B_{t}$ for at most two distinct $t \in V(T)$. The simple treewidth of $G$, denoted by $\operatorname{stw}(G)$, is the least integer $k$ such that $G$ has a $k$-simple tree-decomposition. 


\section{IMPROVED BOUNDS FOR CENTERED COLORINGS AUTHOR}

The notion of simple treewidth was proposed by Knauer and Ueckerdt [10], where graphs of $\operatorname{stw}(G)$ are defined as subgraphs of simple $k$-trees. Since we do not want to discuss $k$-trees and their construction order we stick to our equivalent definition, see [25] for further discussion. It is easy to see that $\operatorname{tw}(G) \leq$ $\operatorname{stw}(G) \leq \operatorname{tw}(G)+1$, for every graph $G$. Graphs of simple treewidth at most 1 are disjoint unions of paths. Graphs of simple treewidth at most 2 are outerplanar graphs. Graphs of simple treewidth at most 3 are planar graphs of treewidth at most 3, i.e., subgraphs of stacked triangulations, see [11].

The first thing to learn about simple treewidth is that it is monotone under taking minors, i.e., if $H$ is a minor of $G$, then $\operatorname{stw}(H) \leq \operatorname{stw}(G)$, see [9]. The next key fact is that the simple treewidth goes down when we restrict the graph to a single BFS layer.

Lemma 16 ([9]). Let $G$ be a connected graph with $\operatorname{stw}(G)=k \geq 1$ and let $\left(V_{0}, V_{1}, \ldots\right)$ be a BFS-layering of $G$. Then $\operatorname{stw}\left(G\left[V_{i}\right]\right) \leq k-1$ for every $i \geq 0$.

For $k \geq 2$ and $p \geq 1$, let $f_{k}(p)$ be the maximum $p$-centered chromatic number of graphs of simple treewidth at most $k$. We will show that $f_{k+1}(p) \leq(p+1) f_{k}(p)$. Given that $f_{2}(p) \in \mathcal{O}(p \log p)$ this yields $f_{k}(p) \in \mathcal{O}\left(p^{k-1} \log p\right)$ for all $k \geq 2$. Below we proceed with the induction step for $k \geq 3$.

Let $G$ be a graph and $\operatorname{stw}(G) \leq k$. Fix a tree-decomposition $(T, \mathcal{B})$ of $G$ witnessing the simple treewidth and let $G^{+}$be a supergraph of $G$ so that each $B \in \mathcal{B}$ induces a clique in $G^{+}$. Clearly, $\operatorname{stw}\left(G^{+}\right) \leq k$ and $G^{+}$is chordal. Since every $p$-centered coloring of $G^{+}$is a $p$-centered coloring of $G$ we work with $G^{+}$. Let $\left(V_{0}, V_{1}, \ldots\right)$ be a BFS layering of $G^{+}$.

Let $v$ be a vertex in $G^{+}$with $v \in V_{i}$. We define $\alpha(v)=i \bmod (p+1)$. By Lemma $16 \operatorname{stw}\left(G^{+}\left[V_{i}\right]\right) \leq$ $k-1$. Let $\beta_{i}$ be a $p$-centered coloring of $G^{+}\left[V_{i}\right]$ using at most $f_{k-1}(p)$ colors. We define $\beta(v)=\beta_{i}(v)$. Finally, for a vertex $v \in V\left(G^{+}\right)$we define $\phi(v)=(\alpha(v), \beta(v))$. Clearly, $\phi$ uses at most $(p+1) \cdot f_{k-1}(p)$ colors. We claim that $\phi$ is a $p$-centered coloring of $G^{+}$.

Let $H$ be a connected subgraph of $G^{+}$. We want to show that either $\phi$ uses more than $p$ colors on $H$ or there is a color that appears exactly once on $H$. Let $\ell$ be minimal such that $V(H) \cap V_{\ell} \neq \emptyset$. The set $X=V(H) \cap V_{\ell}$ induces a connected subgraph of $G^{+}$by Corollary 15 (here we are using that $G^{+}$is chordal). Since $\beta_{\ell}$ is a $p$-centered coloring of $G^{+}\left[V_{\ell}\right]$ we have that either $|\beta(X)|>p$ or there is a vertex in $X$ of unique color under $\beta$. In the first case, $\phi$ takes more than $p$ values on $V(H)$. In the second case, fix the vertex $x \in X$ of the unique color under $\beta$. If $x$ has a unique color under $\phi$ in $H$, then we are done. Otherwise, let $x^{\prime} \in V(H)$ be a vertex with $x \neq x^{\prime}$ and $\phi(x)=\phi\left(x^{\prime}\right)$. Let $x^{\prime} \in V_{\ell^{\prime}}$. Since $x$ has a unique color in $V(H) \cap V_{\ell}$, we get that $\ell \neq \ell^{\prime}$. Since $\alpha(x)=\alpha\left(x^{\prime}\right)$ we conclude that $\ell^{\prime}-\ell \geq p+1$. Since $x$ and $x^{\prime}$ are two vertices in a connected graph $H$ we know that $H$ must intersect every layer $V_{k}$ with $\ell \leq k \leq \ell^{\prime}$. This means that $\alpha$ takes all possible $p+1$ values on the vertices of $H$. Therefore, $\phi$ uses at least $p+1$ colors on $H$, as desired.

The proof implies an algorithm for coloring simple $k$-trees. This algorithm may have many calls for the corresponding algorithm for $(k-1)$-trees but, since the graphs of these calls are disjoint, the overall time complexity remains $\mathcal{O}(n)$ for fixed $k$.

\section{Lower bound for graphs of bounded treewidth}

Let $p$ be a positive integer. A vertex coloring $\phi$ of a graph $G$ is $p$-linear if for every path $P$ in $G$ either $\phi$ uses more than $\phi$ colors on $P$ or there is a color that appears exactly once on $P$. The p-linear chromatic 
number $\operatorname{lin}_{p}(G)$ of $G$ is the minimum integer $k$ such that there is a $p$-linear coloring of $G$ using $k$ colors.

Clearly, every $p$-centered coloring is a $p$-linear coloring. Thus, $\chi_{p}(G) \leq \operatorname{lin}_{p}(G)$ for every graph $G$. We show below that for every $p \geq 0$ and $t \geq 0$, there is a graph $G$ of treewidth at most $t$ with

$$
\operatorname{lin}_{p}(G) \geq\left(\begin{array}{c}
p+t \\
t
\end{array}\right)
$$

We start with the key definition for our inductive construction. For an integer $x$ with $x \geq 1$, a rooted tree $R$ is $x$-ary, if every non-leaf vertex of $R$ has degree at least $x$. The depth of a vertex $v$ in a rooted tree $R$ is the number of vertices on the path between $v$ and the root in $R$. A rooted tree $R$ has depth $d$ if every leaf in $R$ has depth $d$. A subgraph $R$ of a graph $G$ is $(p, d, x)$-brushed if:

(i) $R$ is an $x$-ary tree of depth $d$; and

(ii) for every vertex $v$ in $R$, if $i \in[d]$ is the depth of $v$ in $R$, then there exist indices $j_{1}, \ldots, j_{k}$ with $1 \leq k \leq p$ and $i=j_{1}<\cdots<j_{k}=d$ such that for every leaf $w$ of $R$ that lies in the subtree of $v$, if $u_{i} \cdots u_{d}$ is a path in $R$ connecting $u=u_{i}$ with $w=u_{d}$, then the sequence $u_{j_{1}} \cdots u_{j_{k}}$ induce a path in $G$.

We construct a family of graphs $\left\{G_{(p, t, x, N)} \mid p \geq 0, t \geq 0, x \geq 2, N \geq 1\right\}$ such that the following invariants hold:

1. $\operatorname{tw}\left(G_{(p, t, x, N)}\right) \leq t$; and

2. for every integer $p^{\prime}$ with $p^{\prime} \geq p$ and every $p^{\prime}$-linear coloring $\phi$ of $G_{(p, t, x, N)}$ either:

(a) $\phi$ uses at least $N$ colors on $G_{(p, t, x, N)}$, or

(b) $G$ has a $\left(p,\left(\begin{array}{c}p+t \\ t\end{array}\right), x\right)$-brushed subgraph $R$ and there is a sequence $\left(\alpha_{1}, \ldots, \alpha_{\left(\begin{array}{c}p+t \\ t\end{array}\right)}\right)$ of distinct colors so that for every $i \in\left\{1, \ldots,\left(\begin{array}{c}p+t \\ t\end{array}\right)\right\}$ and every vertex $v$ of depth $i$ in $R$, we have $\phi(v)=\alpha_{i}$.

This family, in particular $G_{\left(p, t, 2,\left(\begin{array}{c}p+t \\ t\end{array}\right)\right)}$, will witness the statement of the theorem.

We start the construction with the base cases. Let $x \geq 2$ and $N \geq 1$. For every $p \geq 0$ and every $t \geq 0$ the graphs $G_{(0, t, x, N)}$ and $G_{(p, 0, x, N)}$ contain just a single vertex. Since these graphs have no edges, their treewidth is $0 \leq t$, so invariant (1) holds. A single vertex is also an $x$-ary tree of depth 1 , so invariant (2b) holds.

For the inductive step let $p \geq 1, t \geq 1, x \geq 2$, and $N \geq 1$. Let $M=\left(\begin{array}{c}p+t-1 \\ t-1\end{array}\right)$ and $X=(x-1) N^{M}+1$. The graph $G_{(p, t, x, N)}$ is obtained from a copy $G^{0}$ of $G_{(p-1, t, X, N)}$ by adding for every vertex $v$ of $G^{0}$ disjoint copies $G^{v, 1}, \ldots, G^{v, X}$ of $G_{(p, t-1, x, N)}$ in such a way that each vertex of $G^{v, i}$ with $i \in[X]$ is adjacent to $v$ and to no other vertices outside $G^{v, i}$.

First, we argue that $\operatorname{tw}\left(G_{(p, t, x, N)}\right) \leq t$. Take a tree-decomposition $\left(T^{0}, \mathcal{B}^{0}\right)$ of $G^{0}$ of width $\leq t$ and for each $v \in V\left(G^{0}\right)$ and each $i \in[X]$ take a tree-decomposition $\left(T^{v, i}, \mathcal{B}^{v, i}\right)$ of $G^{v, i}$ of width $\leq t-1$. We construct a tree-decomposition $(T, \mathcal{B})$ of $G_{(p, t, x, N)}$ as follows. Let $V(T)=V\left(T^{0}\right) \cup \cup V\left(T^{v, i}\right)$. The edges of $T$ include all the edges of $T^{0}$ and all the edges of $T^{v, i}$ for every $v \in V\left(G^{0}\right)$ and $i \in[X]$. Additionally, for every $v \in V\left(G^{0}\right)$, fix some $t_{v} \in V(T)$ such that $v \in B_{t_{v}}^{0} \in \mathcal{B}^{0}$ and for each $i$ fix some vertex $t^{v, i}$ of $T^{v, i}$. In $T$ the tree $T$ is connected to $T^{v, i}$ with the extra edge $t_{v} t^{v, i}$. In simple words, we make a tree out of the 


\section{IMPROVED BOUNDS FOR CENTERED COLORINGS AUTHOR}

forest formed by previous trees. The set $\mathcal{B}$ of bags for $T$ are defined as follows: for every $t \in V\left(T^{0}\right)$ let $B_{t}=B_{t}^{0}$ where $B_{t}^{0} \in \mathcal{B}^{0}$, and for every $t \in V\left(T^{v, i}\right)$ let $B_{t}=B_{t}^{v, i} \cup\{v\}$. It is elementary to verify that $(T, \mathcal{B})$ is a tree-decomposition of $G_{(p, t, x, N)}$ and the width of $(T, \mathcal{B})$ is at most $t$.

Let $p^{\prime} \geq p$ and let $\phi$ be a $p^{\prime}$-linear coloring of $G_{(p, t, x, N)}$. We want to show that (2) is satisfied for the coloring $\phi$. First of all, if $\phi$ uses at least $N$ colors, then (2a) holds. Therefore from now on we assume that $\phi$ uses less than $N$ colors.

Since $G^{0}$ is isomorphic to $G_{(p-1, t, X, N)}$ we have that $G^{0}$ has a $\left(p,\left(\begin{array}{c}p-1+t \\ t\end{array}\right), X\right)$-brushed subgraph that satisifes (2b). We fix a witnessing $X$-ary tree $R^{0}$ of depth $\left(\begin{array}{c}p-1+t \\ t\end{array}\right)$ and the respective sequence of colors $C^{0}=\left(\alpha_{1}, \ldots, \alpha_{\left(\begin{array}{c}p-1+t \\ t\end{array}\right)}\right)$ such that $\phi(u)=\alpha_{i}$, for every vertex $u$ of depth $i$ in $R^{0}$.

Let $u$ be a leaf of $R^{0}$ and $j \in[X]$. We claim that no vertex in $G^{u, j}$ is colored by $\phi$ with a color from $C^{0}$. In order to get a contradiction, suppose that $\phi(v)=\alpha_{i}$ for some $v$ in $G^{u, j}$ and $i \in\left[\left(\begin{array}{c}p-1+t \\ t\end{array}\right)\right]$. If $i=\left(\begin{array}{c}p-1+t \\ t\end{array}\right)$, then $\phi(u)=\alpha_{i}=\phi(v)$, while $u$ and $v$ are adjacent in $G_{(p, t, x, N)}$. Since $p^{\prime} \geq p \geq 1$, the connected subgraph of $G_{(p, t, x, N)}$ induced by $\{u, v\}$ contradicts the fact that $\phi$ is $p^{\prime}$-linear. Now assume that $1 \leq i<\left(\begin{array}{c}p-1+t \\ t\end{array}\right)$. Let $u_{i}$ and $u_{i+1}$ be the ancestors of $u$ in $R^{0}$ of depth $i$ and $i+1$, respectively; $\left(u_{i+1}=u\right.$ is possible). Since $X \geq 2, u_{i}$ has some child $u^{\prime}$ distinct from $u_{i+1}$. Let $u^{\prime \prime}$ be a leaf (vertex of depth $\left(\begin{array}{c}p-1+t \\ t\end{array}\right)$ ) of $R^{0}$ in the subtree of $u^{\prime}$. Consider the paths $u_{i} \cdots u_{\left(\begin{array}{c}p-1+t \\ t\end{array}\right)}$ and $w_{i} \cdots w_{\left(\begin{array}{c}p-1+t \\ t\end{array}\right)}$ in $R$ where $u_{\left(\begin{array}{c}p-1+t \\ t\end{array}\right)}=u$, $w_{i}=u_{i}, w_{i+1}=u^{\prime}$, and $w_{\left(\begin{array}{c}p-1+t \\ t\end{array}\right)}=u^{\prime \prime}$. Since $R^{0}$ is $\left(p,\left(\begin{array}{c}p-1+t \\ t\end{array}\right), X\right)$-brushed in $G^{0}$ there exist indices $j_{1}, \ldots, j_{k}$ with $1 \leq k \leq p-1$ and $i=j_{1}<j_{2}<\cdots<j_{k}=\left(\begin{array}{c}p-1+t \\ t\end{array}\right)$ such that $u_{j_{1}} \cdots u_{j_{k}}$ and $w_{j_{1}} \cdots w_{j_{k}}$ are paths in $G^{0}$ connecting $u_{i}=u_{j_{1}}$ with $u=u_{j_{k}}$ and $u_{i}=w_{j_{1}}$ with $u^{\prime \prime}=w_{j_{k}}$, respectively. Consider the path $P=u_{j_{k}} \cdots u_{j_{1}} w_{j_{2}} \cdots w_{j_{k}}$ connecting $u=u_{j_{k}}$ and $u^{\prime \prime}=w_{j_{k}}$ in $G^{0}$ and recall that $\phi\left(u_{j_{\ell}}\right)=\alpha_{j_{\ell}}=\phi\left(w_{j_{\ell}}\right)$, for all $\ell \in[k]$. Thus, $\phi$ uses exactly $k$ colors on $P$ and the only unique color on $P$ is $\phi\left(u_{i}\right)=\alpha_{i}$. Now since $v$ and $u_{j_{k}}=u$ are adjacent in $G_{(p, t, x, N)}$, the following sequence is a path in $G_{(p, t, x, N)}$ :

$$
v u_{j_{k}} \cdots u_{j_{1}} w_{j_{2}} \cdots w_{j_{k}} \text {. }
$$

Since $\phi(v)=\alpha_{i}=\phi\left(u_{i}\right)$, there is no color used by $\phi$ exactly once on that path. Furthermore, $\phi$ uses exactly $k \leq p-1 \leq p^{\prime}$ colors on that path. This contradicts the fact that $\phi$ is $p^{\prime}$-linear coloring of $G_{(p, t, x, N)}$. Thus, no vertex in $G^{u, j}$ is colored by $\phi$ with a color from $C^{0}$

Let $u$ be a leaf of $R^{0}$ and $j \in[X]$. Since $G^{u, j}$ is isomorphic to $G_{(p, t-1, x, N)}$, we have that $G^{u, j}$ has a $(p, M, x)$-brushed subgraph $R^{u, j}$ that satisifes (2b). We fix a witnessing $x$-ary tree $R^{u, j}$ of depth $M$ and the respective sequence of colors $C^{u, j}=\left(\beta_{1}^{u, j}, \ldots, \beta_{M}^{u, j}\right)$ such that $\phi(v)=\beta_{k}^{u, j}$, for every $k \in[M]$ and every vertex $v$ of depth $k$ in $R^{u, j}$. It follows from the previous paragraph that $\alpha_{i} \neq \beta_{k}^{u, j}$, for all $i \in\left[\left(\begin{array}{c}p-1+t \\ t\end{array}\right)\right]$ and $k \in[M]$.

Since $\phi$ takes less than $N$ values, there are less then $N^{M}$ possibilities for the color sequence $C^{u, j}$. Since $X=(x-1) N^{M}+1$, we find $x$ values $j \in[X]$ such that the color sequences $C^{u, j}$ are identical, we let $C^{u}=\left(\beta_{1}^{u}, \ldots, \beta_{M}^{u}\right)$ be this repeated color sequence.

We now perform a bottom-up traversal on $R^{0}$. We start by marking all the leaves as being visited. When reaching an internal vertex $v$ of $R^{0}$ its children $u_{1}, \ldots, u_{X}$ have been visited and each of them has an associated color sequence $C^{u_{j}}$. Again, since $X=(x-1) N^{M}+1$, we find $x$ values $j \in[X]$ such that the color sequences $C^{u_{j}}$ are identical, we define $C^{v}$ to be this repeated color sequence and mark $v$ as visited. 
When all vertices have been maked visited every internal vertex $v$ in $R_{0}$ has $x$ children $u_{1}, \ldots, u_{x}$ such that $C^{v}=C^{u_{i}}$ for all $i \in[x]$. This way starting from the root of $R^{0}$ we can filter out a subtree $R^{\prime}$ of $R^{0}$ such that $R^{\prime}$ is an $x$-ary tree of depth $\left(\begin{array}{c}p-1+t \\ t\end{array}\right)$ rooted at the root of $R_{0}$ with the properties that

(i) for every vertex $v$ in $R^{\prime}, v$ has the same depth in $R^{\prime}$ as in $R^{0}$; and

(ii) there is a sequence $C=\left(\beta_{1}, \ldots, \beta_{M}\right)$ such that $C^{v}=C$ for all $v$ in $R^{\prime}$.

Finally, we define a tree $R$ as a tree obtained from $R^{\prime}$ by attaching to each leaf $u$ of $R^{\prime}$ exactly $x$ trees among $R^{u, j}$ for $j \in[X]$ such that $C^{u, j}=C$. Therefore $R$ is an $x$-ary tree. Note also that the depth of $R$ is the sum of the depth of $R^{\prime}$ and $M=\left(\begin{array}{c}p+t-1 \\ t-1\end{array}\right)$ (which is the depth of each $R^{u, j}$ ). Thus, the depth of $R$ is

$$
\left(\begin{array}{c}
p-1+t \\
t
\end{array}\right)+\left(\begin{array}{c}
p+t-1 \\
t-1
\end{array}\right)=\left(\begin{array}{c}
p+t \\
t
\end{array}\right) .
$$

We claim that $R$ is $\left(p,\left(\begin{array}{c}p+t \\ t\end{array}\right), x\right)$-brushed in $G_{(p, t, x, N)}$. To prove this, we need to verify the item (ii) of the definition. Let $v$ be a vertex in $R$ and $i$ be the depth of $v$ in $R$.

If $i>\left(\begin{array}{c}p-1+t \\ t\end{array}\right)$, then $v$ lies in one of the trees attached to $R^{\prime}$ in the construction of $R$, say $v$ is in $R^{u, j}$ for some leaf $u$ of $R^{0}$ and $j \in[X]$. Clearly, the depth of $v$ in $R^{u, j}$ is $i^{\prime}=i-\left(\begin{array}{c}p-1+t \\ t\end{array}\right)$. Since $R^{u, j}$ is $(p, M, x)$-brushed in $G^{u, j}$, we get the indices $j_{1}, \ldots, j_{k}$ with $1 \leq k \leq p$ and $i^{\prime}=j_{1}<\cdots<j_{k}=M$ such that for every leaf $w$ of $R^{u, j}$ that lies in the subtree of $v$, if $u_{i^{\prime}} \cdots u_{M}$ is a path in $R^{u, j}$ connecting $v=u_{i^{\prime}}$ with $w=u_{M}$, then the sequence $u_{j_{1}} \cdots u_{j_{k}}$ induce a path in $G^{u, j}$. Thus, in this case (ii) holds for $v$.

If $i \leq\left(\begin{array}{c}p-1+t \\ t\end{array}\right)$, then $v$ lies in $R^{\prime}$. Since $R^{0}$ is $\left(p-1,\left(\begin{array}{c}p-1+t \\ t\end{array}\right), X\right)$-brushed in $G^{0}$, we get the indices $j_{1}, \ldots, j_{k}$ with $1 \leq k \leq p-1$ and $i=j_{1}<\cdots<j_{k}=\left(\begin{array}{c}p-1+t \\ t\end{array}\right)$ such that for every leaf $w$ of $R^{0}$ that lies in the subtree of $v$, if $u_{i} \cdots u_{\left(\begin{array}{c}p-1+t \\ t\end{array}\right)}$ is a path in $R^{0}$ connecting $u=u_{i}$ with $w=u_{\left(\begin{array}{c}p-1+t \\ t\end{array}\right)}$, then the sequence $u_{j_{1}} \cdots u_{j_{k}}$ induces a path in $G^{0}$. Consider a sequence $j_{1}, \ldots, j_{k}$ extended with $j_{k+1}=\left(\begin{array}{c}p+t \\ t\end{array}\right)$. Note that its length is $k+1 \leq p$. Now consider any leaf $w^{\prime}$ of $R$ that lies in the subtree of $v$. Let $w$ be the leaf of $R^{0}$ such that $w^{\prime}$ lies in $R^{w, j}$ for some $j \in[X]$. Let $u_{i} \cdots u_{\left(\begin{array}{c}p+t \\ t\end{array}\right)}$ be the path in $R$ between $u_{i}=v$ and $u_{\left(\begin{array}{c}p+t \\ t\end{array}\right)}=w^{\prime}$. We claim that $u_{j_{1}} \cdots u_{j_{k+1}}$ is a path in $G_{(p, t, x, N)}$. Indeed, $u_{j_{1}} \cdots u_{j_{k}}$ is a path in $G^{0}$ and $u_{j_{k}}=w$ is adjacent with $u_{j_{k+1}}=w^{\prime}$ in $G^{u, j}$. This completes the proof that $R$ is $\left(p,\left(\begin{array}{c}p+t \\ t\end{array}\right), x\right)$-brushed in $G_{(p, t, x, N)}$.

It remains to argue that the item (2b) holds for the coloring $\phi$ and the subgraph $R$ in $G_{(p, t, x, N)}$. Consider the concatenated color sequence

$$
\left(\alpha_{1}, \ldots, \alpha_{\left(\begin{array}{c}
p-1+t \\
t
\end{array}\right)}, \beta_{1}, \ldots, \beta_{M}\right)
$$

Let $i \in\left[\left(\begin{array}{c}p+t \\ t\end{array}\right)\right]$ and let $v$ be a vertex of depth $i$ in $R$. If $i \leq\left(\begin{array}{c}p-1+t \\ t\end{array}\right)$, then $v$ is a vertex in $R^{0}$ and as such $\phi(v)=\alpha_{i}$. If $i>\left(\begin{array}{c}p-1+t \\ t\end{array}\right)$, then $v$ is a vertex in $R^{u, j}$ for some $u$ leaf of $R^{0}$ and $j \in[X]$. Since $R^{u, j}$ was chosen to attach to $R^{\prime}$ in the construction of $R$, we have $C^{u, j}=C$. Therefore, $\phi(v)=\beta_{i-\left(\begin{array}{c}p-1+t \\ t\end{array}\right)}$, as required.

Therefore, $G_{(p, t, x, N)}$ satisfies both invariants. This completes the proof.

\section{Lower bounds for graphs of bounded simple treewidth}

In this section we prove Theorem 7.(ii). We first deal with the case $k=2$, i.e., with the case of outerplanar graphs, which was independently stated as Theorem 6.(ii). We then generalize the proof for the case of larger $k$. The case $k=3$ yields Theorem 6.(iv). 
IMPROVED BOUNDS FOR CENTERED COLORINGS AUTHOR

\subsection{Proof of the lower bound for outerplanar graphs}

\section{Constructing the family of graphs}

The tree of fans $F(w, d)$ is obtained from a rooted complete $w$-ary tree of depth $d$ by connecting the children of each non-leaf vertex with a path. Note that $F(w, d)$ is outerplanar, the vertices are partitioned into $d+1$ levels. Level 0 consists of the root vertex and level $j$ has $w^{j}$ vertices, hence there are $\sum_{j=0}^{d} w^{j}$ vertices in total.

Let $s=\lfloor p / 2\rfloor$ and $t=\lfloor s / 2\rfloor+1$ and $f_{2}(p)=t\lfloor\log s\rfloor$. We let $H$ be a tree of fans $F\left(s f_{2}(p), t\right)$ and $\phi$ be a $p$-centered coloring of $H$. We claim that $\phi$ uses at least $f_{2}(p)$ colors.

\section{A clean subgraph of $H$}

We identify a clean subgraph $H^{*}$ of $H$ isomorphic to $F(s, t)$. The cleaning is done top-down. The root $v_{0}$ is clean by definition. If a vertex $v$ is identified as clean we look at the coloring of the path $P_{v}$ on its children. Let $U_{v}$ be the set of unique colors, i.e., the set of colors which occur only once on $P_{v}$. If $\left|U_{v}\right| \geq f_{2}(p)$ we have a proof that $\phi$ uses at least $f_{2}(p)$ colors. Otherwise considering the length of $P_{v}$ which is $s f_{2}(p)$ and the bound $\left|U_{v}\right|<f_{2}(p)$ we conclude that $P_{v}$ contains a subpath of size $s$ which contains no vertex with a unique color, i.e., every color that appears on the subpath is assigned to at least two vertices of $P_{v}$. Fix such a subpath $Q_{v}$ and declare its vertices to be clean. Then $H^{*}$ is simply the subgraph of $H$ induced by the clean vertices.

\section{The spine for the master caterpillar of $H^{*}$}

A caterpillar rooted at $w_{0}$ of depth $d$ in $H$ consists of a spine, this is a path $w_{0}, w_{1}, \ldots w_{d}$ such that $w_{i+1}$ is a child of $w_{i}$, additionally there may be hairs, they are leaves attached to vertices of the spine. We require that hairs attached to a spine vertex $w$ are children of $w$, moreover, $w_{d}$ has no attached hairs, whence, we also count it as a hair attached to $w_{d-1}$. A caterpillar in $H$ is $H^{*}$-based if all spine vertices belong to $H^{*}$.

Starting from the root $v_{0}$ of $H^{*}$ we are going to identify a path $S=v_{0}, v_{1}, \ldots, v_{t}$ in $H^{*}$ such that $v_{i}$ is a vertex of level $i$. Later we will see that there is a caterpillar $K_{0}$ in $H$ with spine $S$ such that $\phi$ uses at least $f_{2}(p)$ colors on $K_{0}$.

Suppose the spine vertex $v_{\ell-1}$ has been identified. We now describe a procedure to find $v_{\ell}$. Let $Q$ be the path of clean children of $v_{\ell-1}$ in $H^{*}$, this path consists of $s$ vertices. With iterated halving we identify a sequence of nested intervals $Q=Q_{0} \supset Q_{1} \supset \cdots$ which closes in at a single vertex $v_{\ell}$. The iteration requires at least $\lfloor\log s\rfloor$ steps. When $Q_{j}$ has been identified let $U_{j}$ be the set of unique colors on $Q_{j}$. Since $\left|Q_{j}\right| \leq s \leq p$ and $\phi$ is $p$-centered $U_{j} \neq \emptyset$. Divide $Q_{j}$ into its left and right half $Q_{j}^{(\ell)}$ and $Q_{j}^{(r)}$, such that 
||$Q_{j}^{(\ell)}|-| Q_{j}^{(r)}|| \leq 1$. Let $U_{j}^{(\ell)}$ and $U_{j}^{(r)}$ be the subsets of those colors of $U_{j}$ which appear in $Q_{j}^{(\ell)}$ and $Q_{j}^{(r)}$, respectively. It may happen that one of $U_{j}^{(\ell)}$ and $U_{j}^{(r)}$ is empty.

In the following we let $\sigma \in\{\ell, r\}$ and $\bar{\sigma}$ be such that $\{\sigma, \bar{\sigma}\}=\{\ell, r\}$. Let $d^{(\sigma)}$ be the minimum depth of an $H^{*}$-based caterpillar rooted at a vertex of $Q_{j}^{(\sigma)}$ which contains all the colors of $U_{j}^{(\bar{\sigma})}$. If there is no such caterpillar we let $d^{(\sigma)}=p$.

Claim. $\left(d^{(\ell)}-1\right)+\left(d^{(r)}-1\right)>p-\left|Q_{j}\right|$.

Proof. For $\sigma \in\{\ell, r\}$ let $K^{(\sigma)}$ be an $H^{*}$-based caterpillar of depth $d^{(\sigma)}$ rooted at a vertex of $Q_{j}^{(\sigma)}$ which collects all colors of $U_{j}^{(\bar{\sigma})}$. We assume that every hair in $K^{(\sigma)}$ has a color from $U^{(\bar{\sigma})}$ as otherwise we simply remove it from $K^{(\sigma)}$. Let $\Gamma_{0}$ be the subgraph of $H$ which is obtained as the union of $Q_{j}, K^{(\ell)}$, and $K^{(r)}$. Note that the number of colors used by $\phi$ on $\Gamma_{0}$ is bounded by $\left|Q_{j}\right|+\left(d^{(\ell)}-1\right)+\left(d^{(r)}-1\right)$ as hairs, including the last vertices of the spines, only reuse colors used on $Q_{j}$.

In order to get a contradiction, suppose that $\left(d^{(\ell)}-1\right)+\left(d^{(r)}-1\right) \leq p-\left|Q_{j}\right|$. Then we have that the number of colors used by $\phi$ on $\Gamma_{0}$ is bounded by

$$
\left|Q_{j}\right|+\left(d^{(\ell)}-1\right)+\left(d^{(r)}-1\right) \leq\left|Q_{j}\right|+p-\left|Q_{j}\right|=p .
$$

Next we are going to construct a connected supergraph $\Gamma$ of $\Gamma_{0}$ which contains the same colors as $\Gamma_{0}$, i.e., at most $p$, and has no unique color. Such a $\Gamma$ can not exist because $\phi$ is a $p$-centered coloring of $H$.

Consider a color $\alpha$ that is used exactly once in $\Gamma_{0}$. Since all the colors used exactly once at $Q_{j}$ are repeated in $K^{(\ell)}$ or $K^{(r)}$, the color $\alpha$ must appear in one of the $K^{(\sigma)}$, say $K^{(\ell)}$. Since all the colors of the hairs are also used at $Q_{j}$, the color $\alpha$ must be used at a vertex $v$ of the spine of $K^{(\ell)}$ but not within $Q_{j}$. Therefore the parent of $v$, say $v^{\prime}$, also belongs to $K^{(\ell)}$. Since $K^{(\ell)}$ is $H^{*}$-based, $v$ and $v^{\prime}$ belong to $H^{*}$, i.e., they are clean. Therefore there is a child $v^{\prime \prime}$ of $v^{\prime}$ in $H$ with $v^{\prime \prime} \neq v$ and $\phi\left(v^{\prime \prime}\right)=\phi(v)=\alpha$. We add such a vertex $v^{\prime \prime}$ to $\Gamma$. We apply this procedure for every unique color $\alpha$ of $\Gamma_{0}$.

The resulting graph $\Gamma$ is clearly connected and $|\phi(\Gamma)|=\left|\phi\left(\Gamma_{0}\right)\right| \leq p$ while $\Gamma$ has no unique color under $\phi$. This contradiction completes the proof of the claim.

From the claim we get a $\sigma \in\{\ell, r\}$ with $d^{(\sigma)}-1 \geq \frac{1}{2}\left(p-\left|Q_{j}\right|\right) \geq \frac{1}{2}(p-s) \geq \frac{p}{4} \geq t-1$. Use this $\sigma$ to define $Q_{j+1}=Q_{j}^{(\sigma)}$ and $A_{j+1}=U_{j}^{(\bar{\sigma})}$.

The iterated halving ends with a vertex $v_{\ell}$ and a sequence $A_{1}, A_{2}, \ldots, A_{\lfloor\log s\rfloor}$ of sets of colors. From the construction it follows that $A_{i+1}$ is the set of unique colors of $Q_{i}$ which appear in $Q_{i}^{(\bar{\sigma})}$ while for $j>i+1$ the colors of $A_{j}$ appear in $Q_{i}^{(\sigma)}$. This shows that the sets $A_{1}, A_{2}, \ldots, A_{\lfloor\log s\rfloor}$ are pairwise disjoint. Define $B_{\ell}=\bigcup_{i=1}^{\lfloor\log s\rfloor} A_{i}$. From the definition of $d^{(\sigma)}$ and the inequality $d^{(\sigma)} \geq t$ we can deduce the following observation, which will be crucial:

Every $H^{*}$-based caterpillar of depth at most $t-1$ rooted at $v_{\ell}$ misses at least one color from each $A_{i}$, i.e., it misses at least $\lfloor\log s\rfloor$ colors from $B_{\ell}$. 


\section{IMPROVED BOUNDS FOR CENTERED COLORINGS AUTHOR}

\section{Color collecting sub-caterpillars of the master}

Having defined the vertices $v_{0}, v_{1}, \ldots, v_{t}$ we let $K_{\ell}$ be the caterpillar with spine $v_{\ell}, \ldots, v_{t}$ which includes all the children of $v_{i}$ in $H^{*}$ for each $i=\ell, \ldots, t-1$. With $C_{\ell}$ we denote the set of colors of $K_{\ell}$. Since for $0 \leq j<\ell \leq t$ all vertices of $K_{\ell}$ are also vertices of $K_{j}$ we get $C_{0} \supset C_{1} \supset \cdots \supset C_{t}$. Note that $B_{\ell}$ is a set of colors used by children of $v_{\ell-1}$, therefore, $B_{\ell} \subset C_{\ell-1}$. For $\ell>0$, the caterpillar $K_{\ell}$ is an $H^{*}$-based caterpillar of depth $t-\ell \leq t-1$ rooted at $v_{\ell}$, therefore, $K_{\ell}$ misses at least $\lfloor\log s\rfloor$ colors from $B_{\ell}$. Together this shows that $\left|C_{\ell-1}\right| \geq\left|C_{\ell}\right|+\lfloor\log s\rfloor$, whence $\left|C_{0}\right| \geq t\lfloor\log s\rfloor$.

\subsection{Proof of the lower bound for simple treewidth $\mathrm{k}$}

The proof of the lower bound for graphs of simple treewidth $k$ is based on the same four steps as the proof of the lower bound for outerplanar graphs.

\section{Constructing the family of graphs}

The underlying graph for the lower bound is $G_{k}(w, d)$.

We give a recursive definition: $G_{2}(w, d)=F(w, d)$ where $F(w, d)$ is the tree of fans, the vertices of level $d$ of this tree of fans constitute the boundary $L_{2}$ of $G_{2}(w, d)$. Having defined $G_{j-1}(w, d)$ and its boundary $L_{j-1}$ we aim at defining $G_{j}(w, d)$. We introduce an additional parameter $\delta$ running from 1 to $d$ and graphs $G_{j}(w, d, \delta)$. We then define $G_{j}(w, d)=G_{j}(w, d, d)$.

The graph $G_{j}(w, d, 1)$ is obtained from $G_{j-1}(w, d)$ by making the root $v$ an universal vertex, i.e., for every vertex $u$ of the graph we ensure that the edge $(u, v)$ belongs to the graph. The boundary of $G_{j}(w, d, 1)$ is the boundary $L_{j-1}$ of $G_{j-1}(w, d)$. From $G_{j}(w, d, \delta)$ we obtain $G_{j}(w, d, \delta+1)$ by taking a copy $G(u)$ of $G_{j}(w, d, 1)$ for each $u$ in the boundary and identifying the root of $G(u)$ with $u$. The boundary of $G_{j}(w, d, \delta+1)$ is the union of the boundaries of all the new subgraphs $G(u)$.

The following properties of $G_{k}(w, d)$ will be useful.

(1) $G_{k}(w, d)$ contains a spanning subgraph isomorphic to $F\left(w, d^{k-1}\right)$. Indeed, if we modify the definition slightly by deleting the instruction make the root an universal vertex, then througout the construction we only glue new trees of fans to the boundary vertices of an already constructed tree of fans. The boundary vertices are always the vertices of the largest level. This shows that we obtain a tree of fans. For the parameters use induction and the fact that the depth of the tree of fans underlying $G_{j}(w, d)$ is $d$ times the depth of the tree of fans underlying $G_{j-1}(w, d)$.

(2) The simple treewidth of $G_{j}(w, d)$ is at most one more than the simple treewidth of $G_{j-1}(w, d)$, whence by induction $\operatorname{stw}\left(G_{k}(w, d)\right) \leq k$.

First note that the following argument implies $\operatorname{stw}\left(G_{j}(w, d, 1)\right) \leq \operatorname{stw}\left(G_{j-1}(w, d)\right)+1$. If $\operatorname{stw}(G)=t$ and $G^{+}$is obtained from $G$ by making some vertex $v$ of $G$ an universal vertex, then $\operatorname{stw}(G-v) \leq t$ and $\operatorname{stw}\left(G^{+}\right)=\operatorname{stw}(G-v)+1$.

Since every 2-connected component of $G_{j}(w, d)$ is a copy of $G_{j}(w, d, 1)$ we also have $\operatorname{stw}\left(G_{j}(w, d)\right) \leq$ $\operatorname{stw}\left(G_{j}(w, d, 1)\right)$. 
Let $s=\left\lfloor\frac{p}{k}\right\rfloor$, and $t=\frac{k-1}{k} \frac{p}{2}$ and $h=\left\lfloor\frac{t}{k-1}\right\rfloor+1=\left\lfloor\frac{p}{2 k}\right\rfloor+1$. We also define $f_{k}(p)=h^{k-1}\lfloor\log s\rfloor$.

Let $H$ be a $G_{k}\left(s f_{k}(p), h\right)$ and $\phi$ be a $p$-centered coloring of $H$. We claim that $\phi$ is using at least $f_{k}(p)$ colors.

\section{A clean subgraph of $H$}

In a first step we define a clean subgraph $H^{*}$ of $H$. Let $F^{*}$ be obtained by the cleaning procedure of the previous section applied to the tree of fans $F\left(s f_{k}(p), h^{k-1}\right)$ contained in $H$. Note that $F^{*}$ is a tree of fans $F\left(s, h^{k-1}\right)$. Define $H^{*}$ as the graph induced in $H$ by the clean vertices, i.e., by $V\left(F^{*}\right)$.

\section{The spine for the master caterpillar of $H^{*}$}

We adopt a notion of level in $H$ from the tree of fans $F\left(s f_{k}(p), h^{k-1}\right)$ contained in $H$. Let lev $(u)$ denote the level of vertex $u$, the level of the root is 0 and the level of vertices from the boundary of $H$ is $h^{k-1}$. If $v w$ is an edge of $H$ and $\operatorname{lev}(v)<\operatorname{lev}(w)$ we say that $w$ is a child of $v$.

With this child relation the definition of a caterpillar and an $H^{*}$-based caterpillar are exactly as in the previous subsection.

The next step is to define the spine of the master caterpillar. This path $S=v_{0}, v_{1}, \ldots, v_{h^{k-1}}$ starting from the root $v_{0}$ of $H^{*}$ is computed within the clean spanning tree of fans $F^{*}$ contained in $H^{*}$. The procedure is exactly as before, we only recall the main steps.

Suppose $v_{\ell-1}$ has been identified. The next vertex $v_{\ell}$ of $S$ is determined by iterated halving of the path of clean children of $v_{\ell-1}$ in $F^{*}$. This produces $\lfloor\log s\rfloor$ sets of colors whose union is denoted $B_{\ell}$.

With the same definitions as before we again have $\left(d^{(\ell)}-1\right)+\left(d^{(r)}-1\right)>p-\left|Q_{j}\right|$.

Based on the inequality $s+2 t \leq p$ we get $^{1}$ :

Every $H^{*}$-based caterpillar of depth at most $t$ rooted at $v_{\ell}$ misses at least $\lfloor\log s\rfloor$ colors from $B_{\ell}$.

\section{Color collecting sub-caterpillars of the master}

Having defined the vertices of the path $S$ we define a caterpillar $K_{i}$ for each vertex $v_{i}$ of $S$ as follows. The spine $S_{i}=w_{0}, w_{1}, \ldots, w_{d_{i}}$ of $K_{i}$ is determined on the basis of $S$. For the first vertex we take $w_{0}=v_{i}$. When $w_{0}, \ldots, w_{j}$ have been determined and $w_{j} \neq v_{h^{k-1}}$, then let $w_{j+1}$ be the last child of $w_{j}$ in $S$, i.e, the last vertex $w$ on $S$ such that $w_{j} w$ is an edge of $H^{*}$. With a vertex $w_{j}$ of the spine $S_{i}$ the caterpillar $K_{i}$ also includes all the children of $w_{j}$ in $H^{*}$ as hairs.

Claim. $V\left(K_{i+1}\right) \subset V\left(K_{i}\right)$.

\footnotetext{
${ }^{1}$ The difference to the corresponding statement in the previous subsection comes from the fact that there the definition of $t$ had a +1 which is now shifted to the definition of $h$.
} 


\section{IMPROVED BOUNDS FOR CENTERED COLORINGS AUTHOR}

Proof. From the definition $\operatorname{lev}\left(v_{i}\right)=\operatorname{lev}\left(v_{i+1}\right)-1$. First suppose that $\operatorname{lev}\left(v_{i}\right)$ is not divisible by $h$. Then in the construction of $H$ the vertex $v_{i}$ was never element of a boundary, i.e., it wasn't used as an universal vertex of a subgraph. Therefore, all the children of $v_{i}$ in $H$ and in particular $v_{i+1}$ belong to level lev $\left(v_{i}\right)+1$. The spine $S_{i}$ thus starts with $v_{i}, v_{i+1}$ and by construction $S_{i}=v_{i} S_{i+1}$. This implies the claim in this case.

Now suppose that $\ell \geq 1$ is maximal with the property that $h^{\ell} \operatorname{divides} \operatorname{lev}\left(v_{i}\right)$. In this case $v_{i}$ was made the universal vertex of some copy $G\left(v_{i}\right)$ of $G_{\ell+1}\left(s f_{k}(p), h, 1\right)$ used in the construction. If the spine $S_{i}$ thus starts with $v_{i}, w$, then $\operatorname{lev}(w)=\operatorname{lev}\left(v_{i}\right)+h^{\ell}$, i.e., $w=v_{i+h^{\ell}}$. Moreover, all the elements $v_{j}$ of the spine $S$ which are between $v_{i}$ and $w$ and all their children also belong to $G\left(v_{i}\right)$ and hence as children of $v_{i}$ also belong to $K_{i}$. The previous considerations also imply that $w$ belongs to the spine of $S_{i+1}$. This proves the claim in this case.

Let $C_{i}$ be the set of colors of $K_{i}$. From the previous claim we get the nesting of the colorsets $C_{0} \supset C_{1} \supset \ldots \supset C_{h^{k-1}}$.

From the construction we know that for each vertex $v_{i}$ in $S$ the caterpillar $K_{i}$ is an $H^{*}$-based caterpillar.

Claim. For $i>0$, the depth $d_{i}$ of $K_{i}$ is smaller than $(k-1) h$.

Proof. Let $S_{i}=w_{0}, w_{1}, \ldots w_{d_{i}}$. Clearly $w_{d_{i}}=v_{h^{k-1}}$. Let $j$ be the least index such that $\operatorname{lev}\left(w_{j}\right)$ is divisible by $h^{k-2}$. Then lev $\left(w_{\ell}\right)$ is also divisible by $h^{k-2}$ for all $j<\ell \leq d_{i}$. Therefore, there are at most $h$ vertices $w_{\ell}$ with lev $\left(w_{\ell}\right)$ divisible by $h^{k-2}$ in $S_{i}$.

From the minimality of $j$ it follows that $w_{0}, \ldots w_{j}$ are all taken from the same copy of $G_{k-1}\left(s f_{k}(p), h\right)$ used in the construction of $H$. By induction, the base case is a tree of fans, we can asume that $j<$ $(k-2) h$.

Since $(k-1) h \leq t+1$ we conclude that the depth of $K_{i}$ is at most $t$ for all $i>0$.

The set $B_{i}$ is a set of colors used by children of the predecessor $v_{i-1}$ of $v_{i}$, therefore, $B_{i} \subset C_{i-1}$. Caterpillar $K_{i}$ is $H^{*}$-based, rooted at $v_{i}$, and of depth at most $t$, therefore, $K_{i}$ misses at least $\lfloor\log s\rfloor$ colors from $B_{i}$. Together this shows that $\left|C_{i-1}\right| \geq\left|C_{i}\right|+\lfloor\log s\rfloor$, whence $\left|C_{0}\right| \geq h^{k-1}\lfloor\log s\rfloor$. This completes the proof that $\phi$ uses at least $f_{k}(p)$ colors on $H$.

\section{Further directions}

We finish the paper with our two favourite problems.

Conjecture 17. Planar graphs admit p-centered colorings with $\mathcal{O}\left(p^{2} \log p\right)$ colors.

Question 18. Do outerplanar graphs admit p-linear colorings with $\mathcal{O}(p)$ colors?

The best we know for Question 18 is $\mathcal{O}(p \log p)$ by Theorem 6.(i).

Another line of thought is that our upper bound for bounded degree graphs implies the existence of $p$-centered colorings with $\mathcal{O}(p)$ colors of planar grids. Interestingly, the authors have not been able to provide a direct construction for such a coloring. 


\section{Acknowledgements}

We are grateful to Michał Pilipczuk for pointing out that the upper bound for bounded degree graphs was the only missing piece to get a polynomial bound for graphs excluding a fixed topological minor. We thank Gwenaël Joret for pointing out how to set up an entropy compression argument for Theorem 3 to get an upper bound $\mathcal{O}\left(p \cdot \Delta^{2-\varepsilon}\right)$ with $\varepsilon=\Omega(1 / p)$. In the earlier version of this paper [1] we have just $\varepsilon>0$. We are also grateful to Hendrik Schrezenmaier, Michał Seweryn, and Raphael Steiner for many lively discussions on $p$-centered colorings.

\section{References}

[1] Michał Dębski, Stefan Felsner, Piotr Micek, and Felix Schröder, Improved bounds for centered colorings, Proceedings of the 2020 ACM-SIAM Symposium on Discrete Algorithms, SODA, 2020, pp. 2212-2226. 1, 7, 26

[2] Loïc Dubois, Gwenaël Joret, Guillem Perarnau, Marcin Pilipczuk, and François Pitois, Two lower bounds for p-centered colorings, Discrete Mathematics \& Theoretical Computer Science 22 (2020), no. 4.7, 7 pages. 4

[3] Vida Dujmović, Gwenaël Joret, Piotr Micek, Pat Morin, Torsten Ueckerdt, and David R. Wood, Planar graphs have bounded queue-number, Journal of the ACM 67 (2020), no. 4.22, 38 pages. 3, 5,6

[4] Vida Dujmović, Pat Morin, and David R. Wood, Layout of graphs with bounded tree-width, SIAM J. Computing 34 (2005), no. 3, 553-579. 14

[5] Zdeněk Dvořák, Daniel Král, and Robin Thomas, Testing first-order properties for subclasses of sparse graphs, Journal of the ACM 60 (2013), no. 5.36, 24 pages. 2

[6] Guillaume Fertin, André Raspaud, and Bruce Reed, Star coloring of graphs, Journal of Graph Theory 47 (2004), no. 3, 163-182. 4

[7] Martin Grohe and Dániel Marx, Structure theorem and isomorphism test for graphs with excluded topological subgraphs, SIAM Journal of Computing 44 (2015), no. 1, 114-159. 4, 12

[8] Jarosław Grytczuk, Jakub Kozik, and Piotr Micek, New approach to nonrepetitive sequences, Random Structures \& Algorithms 42 (2013), no. 2, 214-225. 4

[9] Gwenaël Joret and Piotr Micek, Improved bounds for weak coloring numbers, arXiv:2102.10061, 2021. 17

[10] Kolja Knauer and Torsten Ueckerdt, Simple treewidth, Midsummer Combinatorial Workshop Prague, 2012. 17

[11] Jan Kratochvíl and Michal Vaner, A note on planar partial 3-trees, arXiv:1210.8113, 2012. 4, 13, 17 


\section{IMPROVED BOUNDS FOR CENTERED COLORINGS AUTHOR}

[12] Jeremy Kun, Michael P. O’Brien, Marcin Pilipczuk, and Blair D. Sullivan, Polynomial treedepth bounds in linear colorings, Algorithmica 83 (2021), 361-386. 4

[13] André Kündgen and Michael J. Pelsmajer, Nonrepetitive colorings of graphs of bounded tree-width, Discrete Mathematics 308 (2008), no. 19, 4473 - 4478. 14

[14] Pat Morin, A fast algorithm for the product structure of planar graphs, Algorithmica 83 (2021), no. $5,1544-1558.7$

[15] Robin A. Moser and Gábor Tardos, A constructive proof of the general Lovász Local Lemma, Journal of the ACM 57 (2010), no. 2.11, 15 pages. 4

[16] Jaroslav Nešetřil and Patrice Ossona de Mendez, Grad and classes with bounded expansion. I. Decompositions, European Journal of Combinatorics 29 (2008), no. 3, 760-776. 2

[17] Jaroslav Nešetřil and Patrice Ossona de Mendez, On nowhere dense graphs, European Journal of Combinatorics 32 (2011), no. 4, 600-617. 2

[18] Jaroslav Nešetřil and Patrice Ossona de Mendez, Tree-depth, subgraph coloring and homomorphism bounds, European Journal of Combinatorics 27 (2006), no. 6, 1022 - 1041. 2

[19] Jaroslav Nešetřil, Patrice Ossona de Mendez, and David R. Wood, Characterisations and examples of graph classes with bounded expansion, European Journal of Combinatorics 33 (2012), no. 3, 350 $-373.2$

[20] Michael P. O'Brien and Blair D. Sullivan, An experimental evaluation of a bounded expansion algorithmic pipeline, arXiv:1712.06690, 2017. 3

[21] Michał Pilipczuk and Sebastian Siebertz, Polynomial bounds for centered colorings on proper minor-closed graph classes, Proceedings of the 30. Annual ACM-SIAM Symposium on Discrete Algorithms, SODA, 2019, pp. 1501-1520. 2, 3, 4, 13

[22] Michał Pilipczuk and Marcin Wrochna, On space efficiency of algorithms working on structural decompositions of graphs, ACM Transactions of Computing Theory 9 (2018), no. 4.18, 36 pages. 3

[23] Neil Robertson and P.D Seymour, Graph minors. XVI. Excluding a non-planar graph, Journal of Combinatorial Theory, Series B 89 (2003), no. 1, 43 - 76. 4

[24] Terence Tao, Moser's entropy compression argument, blog post: http://terrytao.wordpress.com/ 2009/08/05/mosers-entropy-compression-argument/, 2009. 4

[25] Lasse Wulf, Stacked treewidth and the Colin de Verdiére number, Bachelorthesis, Karlsruhe Institute of Technology, 2016. 17 


\section{AUTHORS}

Michał Dębski

Faculty of Informatics

Masaryk University

Brno, Czech Republic

and

Faculty of Mathematics and Information Sciences

Warsaw University of Technology

Warsaw, Poland

michal.debski87@gmail.com

Stefan Felsner

Institut für Mathematik

Technische Universität Berlin

Berlin, Germany

felsner@math.tu-berlin.de

Piotr Micek

Institute of Theoretical Computer Science

Faculty of Mathematics and Computer Science

Jagiellonian University

Kraków, Poland

piotr.micek@uj.edu.pl

Felix Schröder

Institut für Mathematik

Technische Universität Berlin

Berlin, Germany

fschroed@math.tu-berlin.de 